\title{
Mathematical Properties of a New Levin-Type Sequence Transformation Introduced by Čížek, Zamastil, and Skála. I. Algebraic Theory
}

\author{
Ernst Joachim Weniger \\ Institut für Physikalische und Theoretische Chemie \\ Universität Regensburg, D-93040 Regensburg, Germany \\ joachim.weniger@chemie.uni-regensburg.de \\ Submitted to Journal of Mathematical Physics \\ Date of Submision: 25 June 2003
}

PACS numbers: 02.30.Lt, 02.30.Mv, 02.60.-x

\begin{abstract}
Č́̌žek, Zamastil, and Skála [J. Math. Phys. 44, 962 - 968 (2003)] introduced in connection with the summation of the divergent perturbation expansion of the hydrogen atom in an external magnetic field a new sequence transformation which uses as input data not only the elements of a sequence $\left\{s_{n}\right\}_{n=0}^{\infty}$ of partial sums, but also explicit estimates $\left\{\omega_{n}\right\}_{n=0}^{\infty}$ for the truncation errors. The explicit incorporation of the information contained in the truncation error estimates makes this and related transformations potentially much more powerful than for instance Padé approximants. Special cases of the new transformation are sequence transformations introduced by Levin [Int. J. Comput. Math. B 3, 371 - 388 (1973)] and Weniger [Comput. Phys. Rep. 10, 189 - 371 (1989), Sections 7 -9; Numer. Algor. 3, 477 - 486 (1992)] and also a variant of Richardson extrapolation [Phil. Trans. Roy. Soc. London A 226, 299 - 349 (1927)]. The algebraic theory of these transformations - explicit expressions, recurrence formulas, explicit expressions in the case of special remainder estimates, and asymptotic order estimates satisfied by rational approximants to power series - is formulated in terms of hitherto unknown mathematical properties of the new transformation introduced by Čížek, Zamastil, and Skála. This leads to a considerable formal simplification and unification.
\end{abstract}




\section{Contents}

\begin{tabular}{lll}
\hline & Introduction & 1
\end{tabular}

2 Explicit Expressions via Annihilation Operators 3

$\begin{array}{lll}3 & \text { Recurrence Formulas } & 9\end{array}$

\begin{tabular}{|lll}
4 & Levin's Explicit Remainder Estimates & 11
\end{tabular}

$\begin{array}{lll}5 & \text { Richardson-Type Transformations } & 19\end{array}$

$\begin{array}{|ll|}6 & \text { Rational Approximants }\end{array}$

\begin{tabular}{lll}
\hline & Summary and Outlook & 30
\end{tabular} 


\section{Introduction}

The most important and most versatile systematic approximation method in quantum physics is eigenvalue perturbation theory (see for example [125]). Thus, the question, whether perturbation expansions converge or diverge, is of principal importance. Already in 1952, Dyson 54 had argued that perturbation expansions in quantum electrodynamics should diverge. Around 1970, Bender and Wu 101112 showed in their work on anharmonic oscillators that factorially divergent perturbation expansions occur also in nonrelativistic quantum mechanics. In the following years, many other quantum systems were investigated, and in the overwhelming majority factorially divergent perturbation expansions were found (see for example [62, Table 1] or the articles reprinted in [89]). Consequently, summation methods are needed to give the divergent perturbation series of quantum physics any meaning beyond mere formal expansions and to extract numerical information from them. A very readable discussion of the usefulness of summation and related techniques from a physicist's point of view can be found in the monograph by Bender and Orszag 8 .

Factorially divergent power series occur also in asymptotic expansions for special functions. However, special functions can normally be computed via a variety of different representations. Accordingly, in mathematics there is usually no compelling need to use divergent series for computational purposes, whereas in quantum physics it is frequently quite difficult or even impossible to find alternatives to divergent perturbation expansions. Consequently, summation techniques are far more important in physics than in mathematics. Nevertheless, the evaluation of special functions by summing divergent asymptotic expansions can be remarkably effective (see for example [133, 136, 140, 147]).

In physics, the best known and most widely used summation techniques are Borel summation [15, 16], which replaces a divergent perturbation expansion by a Laplace-type integral, and the method of Padé approximants [99], which transforms the partial sums of a power series to a rational function. Both approaches have been remarkably successful, but they have - like all other numerical techniques - certain shortcomings and limitations. For example, the Borel method is very powerful, but conceptually and computationally very demanding. From a technical point of view, Padé approximants can be applied remarkably easily, but they are not necessarily powerful enough to sum all perturbation series of interest. For example, Graffi and Grecchi 66] showed rigorously that Padé approximants are not able to sum the perturbation expansion of the octic anharmonic oscillator whose series coefficients grow roughly like $(3 n) ! / n^{1 / 2}$ [11, Eq. (3)]. Accordingly, it is worth while to look for alternative techniques which are at least in some cases capable of producing better summation results.

Padé approximants accomplish an acceleration of convergence or a summation by converting the partial sums of a power series to a doubly indexed sequence of rational functions. This is also done by other, albeit less well known nonlinear transformations (see for example 19, 20,31,152,132). It is not so well known among non-specialists that some of these transformations sum many strongly divergent power series much more effectively than Padé approximants can do it. Particularly suited for the summation of strongly divergent series is a class of sequence transformations introduced by Levin 90] in 1973. These transformations use as input data not only the elements of a slowly convergent or divergent sequence $\left\{s_{n}\right\}_{n=0}^{\infty}$, whose elements may for instance be the partial sums $s_{n}=\sum_{k=0}^{n} a_{k}$ of an infinite series, but also explicit remainder estimates $\left\{\omega_{n}\right\}_{n=0}^{\infty}$. Several generalizations and extensions of Levin's transformation were derived later, for instance in [132 Sections 7 - 9] or in [134]. Further details as well as the description of several other Levin-type transformations can be found in a recent review by Homeier [74.

The explicit incorporation of the information contained in the remainder estimates $\left\{\omega_{n}\right\}_{n=0}^{\infty}$ makes all Levin-type transformation potentially very powerful (see for example the numerical examples in [126, 127, 132]). In the case of divergent alternating se- 
ries, the so-called delta transformation [132, Eq. (8.4-4)] was found to be particularly use-

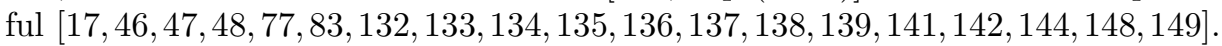

However, sequence transformations in general or the Levin-type transformations mentioned above in special are not only useful for the summation of divergent perturbation expansions. In recent years, many other successful applications of Levin-type transformations have been reported in the literature (see for example $2456,14,13183746475253$.

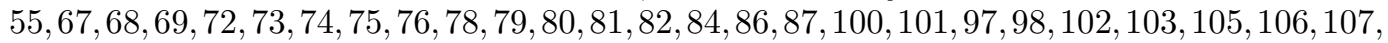
108116117118120128130131132133134135136137138140144147150151 ). This list does not claim to be complete, but it suffices to show that Levin-type transformations are extremely useful computational tools which deserve to be more widely known.

In connection with the summation of the perturbation series for a hydrogen atom in an external magnetic field, Čížek, Zamastil, and Skála introduced a new sequence transformation [48, Eq. (10)], which in the notation of 132 can be expressed as follows:

$$
\mathcal{G}_{k}^{(n)}\left(q_{m}, s_{n}, \omega_{n}\right)=\frac{\sum_{j=0}^{k}(-1)^{j}\left(\begin{array}{l}
k \\
j
\end{array}\right) \prod_{m=1}^{k-1} \frac{\left(n+j+q_{m}\right)}{\left(n+k+q_{m}\right)} \frac{s_{n+j}}{\omega_{n+j}}}{\sum_{j=0}^{k}(-1)^{j}\left(\begin{array}{l}
k \\
j
\end{array}\right) \prod_{m=1}^{k-1} \frac{\left(n+j+q_{m}\right)}{\left(n+k+q_{m}\right)} \frac{1}{\omega_{n+j}}}, \quad k, n \in \mathbb{N}_{0} .
$$

Here as well as later in the text it is always assumed that $\prod_{k=l}^{n} a_{k}=1$ holds if it is a so-called empty product with $l>n$.

The sequence transformation (1.1) contains the unspecified parameters $q_{m}$ with $1 \leq m \leq$ $k-1$. As discussed in Section 2 in more details, several other sequence transformations can be obtained by specifying the parameters $q_{m}$. If we for instance choose $q_{m}=\beta$ with $\beta>0$, we obtain Levin's transformation $\mathcal{L}_{k}^{(n)}\left(\beta, s_{n}, \omega_{n}\right)$ [90] in the notation of [132 7.1-7]), if we choose $q_{m}=\beta+m-1$, we obtain $\mathcal{S}_{k}^{(n)}\left(\beta, s_{n}, \omega_{n}\right)$ [132 Eq. (8.2-7)], which is the parent transformation of the so-called delta transformation [132, Eq. (8.4-4)] mentioned above, and if we choose $q_{m}=\xi-m+1$ with $\xi>0$, we obtain $\mathcal{M}_{k}^{(n)}\left(\xi, s_{n}, \omega_{n}\right)$ [132, Eq. (9.2-6)]. Then, there is a sequence transformation $\mathcal{C}_{k}^{(n)}\left(\alpha, \beta, s_{n}, \omega_{n}\right)$ [134 Eq. (3.2)] which - depending on the value of the parameter $\alpha$ - interpolates between Levin's transformation $\mathcal{L}_{k}^{(n)}\left(\beta, s_{n}, \omega_{n}\right)$ and $\mathcal{S}_{k}^{(n)}\left(\beta, s_{n}, \omega_{n}\right)$. It is obtained by choosing $q_{m}=\beta+[m-1] / \alpha$.

Thus, the transformation $\mathcal{G}_{k}^{(n)}\left(q_{m}, s_{n}, \omega_{n}\right)$ introduced by Č́́žek, Zamastil, and Skála provides a unifying concept for a large and practically important class of sequence transformations, and all results derived for $\mathcal{G}_{k}^{(n)}\left(q_{m}, s_{n}, \omega_{n}\right)$ can immediately be translated to the analogous results for its various special cases. However, so far only the explicit expression for this transformation as the ratio of two finite sums according to (1.1) is known 48, Eq. (10)], and many other mathematical properties of interest are unknown.

In Section 2 the explicit expression for $\mathcal{G}_{k}^{(n)}\left(q_{m}, s_{n}, \omega_{n}\right)$ is rederived by applying a suitable annihilating difference operator to the model sequence (2.12) according to (2.9). This annihilation operator approach was originally introduced in [132 Section 3.2] in connection with a simplified derivation of the explicit expression for Levin's transformation 90. and the construction of explicit expressions for other, closely related sequence transformations [132 Sections 7 - 9]. This annihilation operator approach does not only produce the explicit expression (1.1), but it also provides a convenient starting point for the derivation of a recursive scheme for the numerators and denominators in (1.1), which is done in Section 3. and for a theoretical convergence analysis, which will be done in 146.

In Section 4 simple explicit remainder estimates introduced by Levin 90 and Smith and Ford [126, which in the terminology of 132 yield the $u, t, v$, and $d$ variants of Levin's sequence transformation, are used in combination with $\mathcal{G}_{k}^{(n)}\left(q_{m}, s_{n}, \omega_{n}\right)$. The effectivity of 
these remainder estimates is motivated and studied via some model sequences. Surprisingly, the $v$ type remainder estimate produces more effective asymptotic estimates for the truncation errors of these model sequences than the other simple remainder estimates. Moreover, it is shown that all $t$ type variants considered in this article are actually analogous $d$ type variants in disguise.

In Section 5 variants of $\mathcal{G}_{k}^{(n)}\left(q_{m}, s_{n}, \omega_{n}\right)$ are studied which closely resemble the Richardson extrapolation process [104. These variants be used in the case of logarithmic convergence $(\rho=1$ in (4.7)), whose acceleration constitutes a formidable computational problem.

In Section [6] the $u, t, d$, and $v$ variants of $\mathcal{G}_{k}^{(n)}\left(q_{m}, s_{n}, \omega_{n}\right)$ are applied to the partial sums of a (formal) power series. This produces rational approximants that resemble Padé approximants, which are defined via the accuracy-through-order relationship (6.3). In the case of the $u, t$, and $d$ variants, the resulting rational expressions are actually Padé-type approximants, which satisfy the modified accuracy-through-order relationship (6.15) [22]. In the case of the $v$ variant the resulting expression is a slight generalization of a Padétype approximant. With the help of the accuracy-through-order relationship (6.15), which defines Padé-type approximants, the accuracy-through-order relationships satisfied by these rational functions can be derived easily. These accuracy-through-order relationships are needed if the rational approximants derived from $\mathcal{G}_{k}^{(n)}\left(q_{m}, s_{n}, \omega_{n}\right)$ are to be used for the prediction of unknown power series coefficients.

This article is concluded in Section 7 by a short summary and a critical assessment of the essential features of the new Levin-type transformation introduced by recently Č́žek, Zamastil, and Skála 48.

Only the mathematical properties of $\mathcal{G}_{k}^{(n)}\left(q_{m}, s_{n}, \omega_{n}\right)$ and its various special cases are treated in this article, albeit in a relatively detailed way. Anybody interested in other sequence transformations should consult the monograph by Brezinski and Redivo Zaglia 31 . It contains a wealth of material and provides a very readable introduction to a rapidly growing subfield of numerical mathematics. The older history of sequence transformations up to about 1945 is treated in a monograph by Brezinski [24, and the more recent history is discussed in two articles, also by Brezinski [25, 29.

Finally, one should not forget that the study of sequence transformations remains incomplete without the simultaneous study of Padé approximants. Here, I recommend the book by Baker and Graves-Morris [3].

\section{Explicit Expressions via Annihilation Operators}

Let us assume that $\left\{s_{n}\right\}_{n=0}^{\infty}$ is a slowly convergent or divergent sequence, whose elements may for instance be the partial sums $s_{n}=\sum_{k=0}^{n} a_{k}$ of an infinite series. A sequence transformation is a rule which maps a sequence $\left\{s_{n}\right\}_{n=0}^{\infty}$ to a new sequence $\left\{s_{n}^{\prime}\right\}_{n=0}^{\infty}$ with hopefully better numerical properties.

The basic step for the construction of a sequence transformation is the assumption that the elements of a convergent or divergent sequence $\left\{s_{n}\right\}_{n=0}^{\infty}$ can be partitioned into a (generalized) limit $s$ and a remainder $r_{n}$ according to

$$
s_{n}=s+r_{n}, \quad n \in \mathbb{N}_{0} .
$$

A sequence transformation tries to accomplish an acceleration of convergence or a summation by eliminating the remainders $r_{n}$ as effectively as possible from the input data $s_{n}$ with the help of numerical techniques. In realistic problems, a sequence transformation can only eliminate approximations to the remainders. Consequently, the transformed sequence $\left\{s_{n}^{\prime}\right\}_{n=0}^{\infty}$ will also be of the type of (2.1), which means that $s_{n}^{\prime}$ can also be partitioned into 
the (generalized) limit $s$ and a transformed remainder $r_{n}^{\prime}$ according to

$$
s_{n}^{\prime}=s+r_{n}^{\prime}, \quad n \in \mathbb{N}_{0} .
$$

The transformed remainders $\left\{r_{n}^{\prime}\right\}_{n=0}^{\infty}$ are in general different from zero for all finite values of $n$. However, convergence is accelerated if the transformed remainders $\left\{r_{n}^{\prime}\right\}_{n=0}^{\infty}$ vanish more rapidly than the original remainders $\left\{r_{n}\right\}_{n=0}^{\infty}$ according to

$$
\lim _{n \rightarrow \infty} \frac{s_{n}^{\prime}-s}{s_{n}-s}=\lim _{n \rightarrow \infty} \frac{r_{n}^{\prime}}{r_{n}}=0,
$$

and a divergent sequence is summed if the transformed remainders $r_{n}^{\prime}$ vanish as $n \rightarrow \infty$.

In practice, an in principle unlimited variety of different types of remainders can occur. Therefore, it is essential to make some assumptions - either explicitly or implicitly - which provide the basis for the construction of a sequence transformation.

Let us assume that we have sufficient reason to believe that the elements of a sequence $\left\{s_{n}\right\}_{n=0}^{\infty}$ can for all $n \in \mathbb{N}_{0}$ be expressed by an expansion of the following type:

$$
s_{n}=s+\sum_{j=0}^{\infty} c_{j} \psi_{j}(n)
$$

The $\psi_{j}(n)$ are assumed to be known functions of $n$, but otherwise essentially arbitrary, and the $c_{j}$ are unspecified coefficients independent of $n$. Hence, the ansatz (2.4) incorporates convergent as well as divergent sequences, depending upon the behavior of the functions $\psi_{j}(n)$ as $n \rightarrow \infty$.

If we want to accelerate the convergence of $\left\{s_{n}\right\}_{n=0}^{\infty}$ to its limit $s$ or to sum it in the case of divergence with the help of a sequence transformation, we have to compute approximations to the remainders $\sum_{j=0}^{\infty} c_{j} \psi_{j}(n)$ and to eliminate them from the input data. However, the remainders of the sequence (2.4) contain an infinite number of unspecified coefficients $c_{j}$. Consequently, a complete determination of the remainders and their subsequent elimination cannot be accomplished by purely numerical means.

Let us also assume that the functions $\left\{\psi_{j}(n)\right\}_{j=0}^{\infty}$ form an asymptotic sequence as $n \rightarrow \infty$, i.e, that they satisfy for all $j \in \mathbb{N}_{0}$

$$
\psi_{j+1}(n)=\mathrm{o}\left(\psi_{j}(n)\right), \quad n \rightarrow \infty .
$$

The best, which a purely numerical process can accomplish, is the elimination of a finite number of the leading terms of (2.4). Obviously, this corresponds to the transformation of the sequence (2.4) to a new sequence $\left\{s_{n}^{\prime}\right\}_{n=0}^{\infty}$ whose elements satisfy

$$
s_{n}^{\prime}=s+\sum_{j=0}^{\infty} c_{j}^{\prime} \psi_{k+j}(n), \quad n \in \mathbb{N}_{0}, \quad k \in \mathbb{N} .
$$

The $c_{j}^{\prime}$ are numerical coefficients that depend on $k$ as well as on the coefficients $c_{j}$ in (2.4).

Obviously, the original remainders $\sum_{j=0}^{\infty} c_{j} \psi_{j}(n)$ are not completely eliminated from the elements of the input sequence (2.4). Since, however, the functions $\left\{\psi_{j}(n)\right\}_{j=0}^{\infty}$ are by assumption an asymptotic sequence according to (2.5), the transformed remainders $\sum_{j=0}^{\infty} c_{j}^{\prime} \psi_{k+j}(n)$ in (2.6) should at least for sufficiently large values of $k \in \mathbb{N}$ have significantly better numerical properties than the original remainders $\sum_{j=0}^{\infty} c_{j} \psi_{j}(n)$.

Assumptions about the $n$-dependence of the truncation errors $r_{n}$ can be incorporated into the transformation process via model sequences. In this approach, a sequence transformation is constructed which produces the (generalized) limit $s$ of the model sequence

$$
s_{n}=s+r_{n}=s+\sum_{j=0}^{k-1} c_{j} \psi_{j}(n), \quad k \in \mathbb{N}, \quad n \in \mathbb{N}_{0},
$$


if it is applied to $k+1$ consecutive elements $s_{n}, s_{n+1}, \ldots, s_{n+k}$ of this model sequence. Since the $\psi_{j}(n)$ are assumed to be known functions of $n$, an element of this model sequence contains $k+1$ unknown, the (generalized) limit $s$ and the $k$ unspecified coefficients $c_{0}, c_{1}$, $\ldots, c_{k-1}$. Accordingly, it follows from Cramer's rule that a sequence transformation, which is exact for the model sequence (2.7), can be expressed as the ratio of two determinants (see for example [31 Section 1.5]). However, determinantal representations will not be considered here since they are computationally unattractive. Fortunately, the sequence transformation, which is exact for the general model sequence (2.7), can also be computed recursively, as shown independently by Schneider [119, Håvie [70] and Brezinski [21]. An alternative recursive scheme, which is more economical than the original recursive scheme, was later obtained by Ford and Sidi 63 .

A detailed discussion of the construction of sequence transformations via model sequences as well as many examples can for instance be found in the book by Brezinski and Redivo Zaglia [31] or in [132.

Levin-type sequence transformations try to make the transformation process more efficient by explicitly utilizing the information contained in remainder estimates $\left\{\omega_{n}\right\}_{n=0}^{\infty}$. Thus, a sequence transformation is constructed which is exact for the elements of the model sequence [132, Eq. (3.2-9)]

$$
s_{n}=s+\omega_{n} z_{n}, \quad n \in \mathbb{N}_{0} .
$$

The remainder estimates $\omega_{n}$ are assumed to be known, and the correction terms $z_{n}$ should be chosen in such a way that the products $\omega_{n} z_{n}$ provide sufficiently accurate and rapidly convergent approximations to actual remainders. The principal advantage of this approach is that only the correction terms $\left\{z_{n}\right\}_{n=0}^{\infty}$ have to be determined. If good remainder estimates can be found, the determination of $z_{n}$ and the subsequent elimination of $\omega_{n} z_{n}$ from $s_{n}$ often leads to substantially better results than the construction and subsequent elimination of other approximations to $r_{n}$.

The model sequence (2.8) has another indisputable advantage: There exists a systematic approach for the construction of a sequence transformation which is exact for this model sequence. Let us assume that a linear operator $\hat{T}$ can be found which annihilates for all $n \in$ $\mathbb{N}_{0}$ the correction term $z_{n}$ according to $\hat{T}\left(z_{n}\right)=0$. Then, a sequence transformation, which is exact for the model sequence (2.8), can be obtained by applying $\hat{T}$ to $\left[s_{n}-s\right] / \omega_{n}=z_{n}$. Since $\hat{T}$ annihilates $z_{n}$ and is by assumption linear, the following sequence transformation $\mathcal{T}$ is exact for the model sequence (2.8) [132, Eq. (3.2-11)]:

$$
\mathcal{T}\left(s_{n}, \omega_{n}\right)=\frac{\hat{T}\left(s_{n} / \omega_{n}\right)}{\hat{T}\left(1 / \omega_{n}\right)}=s .
$$

The construction of sequence transformations via annihilation operators was introduced in [132, Section 3.2] in connection with a rederivation of Levin's transformation 90] and the construction of some other, closely related sequence transformations [132 Sections 7 - 9].

Later, this annihilation operator approach was discussed and extended in books by Brezinski [26] and Brezinski and Redivo Zaglia [31] and in articles by Brezinski [25 2728 29], Brezinski and Matos [30, Brezinski and Redivo Zaglia 32,33 34, Brezinski and Salam [35, Homeier [74, Matos [92], and Weniger [134].

Simple and yet very powerful sequence transformations result [132, Sections 7 - 9] if the annihilation operator $\hat{T}$ in (2.9) is based upon the finite difference operator $\Delta$ defined by $\Delta f(n)=f(n+1)-f(n)$. As is well known, the $k$-th power of the finite difference operator annihilates a polynomial $P_{k-1}(n)$ of degree $k-1$ in $n$ according to $\Delta^{k} P_{k-1}(n)=0$. Thus, the correction terms $z_{n}$ in (2.8) should be chosen in such a way that multiplication of $z_{n}$ by some suitable quantity $w_{k}(n)$ yields a polynomial $P_{k-1}(n)$ of degree $k-1$ in $n$. If such a 
$w_{k}(n)$ can be found, then

$$
\Delta^{k}\left[w_{k}(n) z_{n}\right]=\Delta^{k} P_{k-1}(n)=0
$$

and the weighted difference operator $\hat{T}=\Delta^{k} w_{k}(n)$ annihilates $z_{n}$. Thus, the corresponding sequence transformation (2.9) is given by the ratio

$$
\mathcal{T}_{k}^{(n)}\left(w_{k}(n) \mid s_{n}, \omega_{n}\right)=\frac{\Delta^{k}\left[w_{k}(n) s_{n} / \omega_{n}\right]}{\Delta^{k}\left[w_{k}(n) / \omega_{n}\right]} .
$$

The sequence transformation (1.1) introduced by Čížek, Zamastil, and Skála can be constructed on the basis of the model sequence [48, Eq. (9)]

$$
s_{n}=s+\omega_{n} \sum_{j=0}^{k-1} \frac{c_{j}}{\prod_{m=1}^{j}\left(n+q_{m}\right)}, \quad k \in \mathbb{N}, \quad n \in \mathbb{N}_{0} .
$$

It will become clear later (compare (4.5) and the discussion related to it) that in (2.12) as well as in the model sequences (2.16), (2.19), (2.23), and (2.27), which can be derived from (2.12), it makes sense to assume $c_{0} \neq 0$.

Both in the model sequence (2.12) as well as in the sequence transformation, which is derived from it, we want to admit $n=0$. Moreover, this model sequence should have a consistent behavior for all $n \in \mathbb{N}_{0}$. Thus, we normally require $q_{m}>0$ for $0 \leq m \leq k-1$, but otherwise these parameters are essentially arbitrary.

Multiplication of the sum in (2.12) by $\prod_{m=1}^{k-1}\left(n+q_{m}\right)$ yields $\sum_{j=0}^{k-1} c_{j} \prod_{i=j+1}^{k-1}\left(n+q_{m}\right)$, which is a polynomial of degree $k-1$ in $n$. Thus, $\hat{T}=\Delta^{k} \prod_{m=1}^{k-1}\left(n+q_{m}\right)$ is the operator which annihilates the correction term in (2.12), and we obtain from (2.9) the following difference operator representation for the sequence transformation introduced by Č́žek, Zamastil, and Skála [48, Eq. (10)]:

$$
\mathcal{G}_{k}^{(n)}\left(q_{m}, s_{n}, \omega_{n}\right)=\frac{\Delta^{k} \prod_{m=1}^{k-1}\left(n+q_{m}\right) \frac{s_{n}}{\omega_{n}}}{\Delta^{k} \prod_{m=1}^{k-1}\left(n+q_{m}\right) \frac{1}{\omega_{n}}}, \quad k, n \in \mathbb{N}_{0} .
$$

It will become clear later that this difference operator representation is in some sense more fundamental and more important than the explicit expression which can be derived easily from it. We only have to insert the well-known relationship

$$
\Delta^{k} f(n)=(-1)^{k} \sum_{j=0}^{k}(-1)^{j}\left(\begin{array}{l}
k \\
j
\end{array}\right) f(n+j)
$$

into the numerator and denominator of (2.13) to obtain

$$
\mathcal{G}_{k}^{(n)}\left(q_{m}, s_{n}, \omega_{n}\right)=\frac{\sum_{j=0}^{k}(-1)^{j}\left(\begin{array}{l}
k \\
j
\end{array}\right) \prod_{m=1}^{k-1}\left(n+j+q_{m}\right) \frac{s_{n+j}}{\omega_{n+j}}}{\sum_{j=0}^{k}(-1)^{j}\left(\begin{array}{l}
k \\
j
\end{array}\right) \prod_{m=1}^{k-1}\left(n+j+q_{m}\right) \frac{1}{\omega_{n+j}}}, \quad k, n \in \mathbb{N}_{0} .
$$

In the case of large transformation orders $k$, terms that are large in magnitude occur in the binomial sums in the numerator and denominator of (2.15). The same problem occurs 
also in explicit expressions for other Levin-type sequence transformation discussed later. In the case of some FORTRAN compilers, this can easily lead to OVERFLOW. To decrease the magnitude of the terms, it has become customary to include an additional normalization factor. Thus, we divide numerator and denominator of (2.15) by $\prod_{m=1}^{k-1}\left(n+k+q_{m}\right)$ and obtain (1.1). Such an approach is always possible since the coefficients of a ratio like (2.15) are only defined up to a common nonzero factor.

If we choose in (2.12) $q_{m}=\beta$ with $\beta>0$, we obtain the model sequence for Levin's transformation [90] in the notation of [132, Eq. (7.1-2)]:

$$
s_{n}=s+\omega_{n} \sum_{j=0}^{k-1} \frac{c_{j}}{(\beta+n)^{j}}, \quad k \in \mathbb{N}, \quad n \in \mathbb{N}_{0} .
$$

Thus, the corresponding annihilation operator is given by $\hat{T}=\Delta^{k}(\beta+n)^{k-1}$ and Levin's transformation [90] can in the notation of 132 Eq. (7.1-7)] be expressed as follows:

$$
\begin{aligned}
\mathcal{L}_{k}^{(n)}\left(\beta, s_{n}, \omega_{n}\right)= & \mathcal{G}_{k}^{(n)}\left(\beta, s_{n}, \omega_{n}\right)=\frac{\Delta^{k}\left[(\beta+n)^{k-1} s_{n} / \omega_{n}\right]}{\Delta^{k}\left[(\beta+n)^{k-1} / \omega_{n}\right]} \\
= & \frac{\sum_{j=0}^{k}(-1)^{j}\left(\begin{array}{l}
k \\
j
\end{array}\right) \frac{(\beta+n+j)^{k-1}}{(\beta+n+k)^{k-1}} \frac{s_{n+j}}{\omega_{n+j}}}{\sum_{j=0}^{k}(-1)^{j}\left(\begin{array}{l}
k \\
j
\end{array}\right) \frac{(\beta+n+j)^{k-1}}{(\beta+n+k)^{k-1}} \frac{1}{\omega_{n+j}}} \quad k, n \in \mathbb{N}_{0} .
\end{aligned}
$$

If we choose in (2.12) $q_{m}=\beta+m-1$ with $\beta>0$, we obtain the following model sequence [132. Eq. (8.2-1)] for the sequence transformation $\mathcal{S}_{k}^{(n)}\left(\beta, s_{n}, \omega_{n}\right)$ which is a truncated factorial series involving Pochhammer symbols $(\beta+n)_{j}=\Gamma(\beta+n+j) / \Gamma(\beta+n)$ :

$$
s_{n}=s+\omega_{n} \sum_{j=0}^{k-1} \frac{c_{j}}{(\beta+n)_{j}}, \quad k \in \mathbb{N}, \quad n \in \mathbb{N}_{0} .
$$

The fundamental properties of factorial series are for instance discussed in books by Nielsen 94 and Nörlund 95,96.

The annihilation operator for the model sequence (2.19) is given by $\hat{T}=\Delta^{k}(\beta+n)_{k-1}$ and the sequence transformation $\mathcal{S}_{k}^{(n)}\left(\beta, s_{n}, \omega_{n}\right)$, which is exact for the model sequence (2.19), can be expressed as follows [132 Eq. (8.2-7)]:

$$
\begin{aligned}
\mathcal{S}_{k}^{(n)}\left(\beta, s_{n}, \omega_{n}\right)= & \mathcal{G}_{k}^{(n)}\left(\beta+m-1, s_{n}, \omega_{n}\right)=\frac{\Delta^{k}\left[(\beta+n)_{k-1} s_{n} / \omega_{n}\right]}{\Delta^{k}\left[(\beta+n)_{k-1} / \omega_{n}\right]} \\
= & \frac{\sum_{j=0}^{k}(-1)^{j}\left(\begin{array}{l}
k \\
j
\end{array}\right) \frac{(\beta+n+j)_{k-1}}{(\beta+n+k)_{k-1}} \frac{s_{n+j}}{\omega_{n+j}}}{\sum_{j=0}^{k}(-1)^{j}\left(\begin{array}{l}
k \\
j
\end{array}\right) \frac{(\beta+n+j)_{k-1}}{(\beta+n+k)_{k-1}} \frac{1}{\omega_{n+j}}} \quad k, n \in \mathbb{N}_{0} .
\end{aligned}
$$

The ratio (2.21) was originally derived by Sidi [122 for the construction of explicit expressions for Padé approximants of some special hypergeometric series. However, Sidi's article [122] provides no evidence that he intended to use this ratio as a sequence transformation. Moreover, I am not aware of any article of Sidi where the properties of the sequence transformation $\mathcal{S}_{k}^{(n)}\left(\beta, s_{n}, \omega_{n}\right)$ were discussed or where it was applied. Later, $\mathcal{S}_{k}^{(n)}\left(\beta, s_{n}, \omega_{n}\right)$ was apparently used in the master thesis of Shelef [121 for the numerical inversion of Laplace 
transforms, but it seems that the results of this master thesis were not published elsewhere. The first refereed and generally accessible article, where an application of (2.21) as a sequence transformation was described, is [151, and the mathematical properties of (2.21) as a sequence transformation and in particular its connection with factorial series were first discussed in [132, Section 8].

If we choose in (2.12) $q_{m}=\xi-m+1$ with $\xi>0$, we obtain the following model sequence [132, Eq. (9.2-1)] for the sequence transformation $\mathcal{M}_{k}^{(n)}\left(\xi, s_{n}, \omega_{n}\right)$ :

$$
\begin{aligned}
s_{n} & =s+\omega_{n} \sum_{j=0}^{k-1} \frac{c_{j}}{\prod_{m=1}^{j}(\xi+n-m+1)} \\
& =s+\omega_{n} \sum_{j=0}^{k-1} \frac{(-1)^{j} c_{j}}{(-\xi-n)_{j}}=s+\omega_{n} \sum_{j=0}^{k-1} \frac{c_{j}^{\prime}}{(-\xi-n)_{j}}, \quad k \in \mathbb{N}, \quad n \in \mathbb{N}_{0} .
\end{aligned}
$$

Thus, the corresponding annihilation operator is given by $\hat{T}=\Delta^{k}(-\xi-n)_{k-1}$ and the sequence transformation $\mathcal{M}_{k}^{(n)}\left(\xi, s_{n}, \omega_{n}\right)$ can be expressed as follows [132, Eq. (9.2-6)]:

$$
\begin{aligned}
\mathcal{M}_{k}^{(n)}\left(\xi, s_{n}, \omega_{n}\right)= & \mathcal{G}_{k}^{(n)}\left(\xi-m+1, s_{n}, \omega_{n}\right)=\frac{\Delta^{k}\left[(-\xi-n)_{k-1} s_{n} / \omega_{n}\right]}{\Delta^{k}\left[(-\xi-n)_{k-1} / \omega_{n}\right]} \\
= & \frac{\sum_{j=0}^{k}(-1)^{j}\left(\begin{array}{l}
k \\
j
\end{array}\right) \frac{(-\xi-n-j)_{k-1}}{(-\xi-n-k)_{k-1}} \frac{s_{n+j}}{\omega_{n+j}}}{\sum_{j=0}^{k}(-1)^{j}\left(\begin{array}{l}
k \\
j
\end{array}\right) \frac{(-\xi-n-j)_{k-1}}{(-\xi-n-k)_{k-1}} \frac{1}{\omega_{n+j}}} \quad k, n \in \mathbb{N}_{0} .
\end{aligned}
$$

If we choose in 2.12) $q_{m}=\beta+[m-1] / \alpha$ with $\alpha, \beta>0$, we obtain the following model sequence [134 Eq. (3.1)] for the sequence transformation $\mathcal{C}_{k}^{(n)}\left(\alpha, \beta, s_{n}, \omega_{n}\right)$ :

$$
\begin{aligned}
s_{n} & =s+\omega_{n} \sum_{j=0}^{k-1} \frac{c_{j}}{\prod_{m=1}^{j}(\beta+n+[m-1] / \alpha)} \\
& =s+\omega_{n} \sum_{j=0}^{k-1} \frac{\alpha^{j} c_{j}}{(\alpha[\beta+n])_{j}}=s+\omega_{n} \sum_{j=0}^{k-1} \frac{c_{j}^{\prime}}{(\alpha[\beta+n])_{j}}, \quad k \in \mathbb{N}, \quad n \in \mathbb{N}_{0} .
\end{aligned}
$$

Thus, the corresponding annihilation operator is given by $\hat{T}=\Delta^{k}(\alpha[\beta+n])_{k-1}$ and the sequence transformation $\mathcal{C}_{k}^{(n)}\left(\alpha, \beta, s_{n}, \omega_{n}\right)$ can be expressed as follows [134, Eq. (3.2)]:

$$
\begin{aligned}
\mathcal{C}_{k}^{(n)}\left(\alpha, \beta, s_{n}, \omega_{n}\right)= & \mathcal{G}_{k}^{(n)}\left(\beta+[m-1] / \alpha, s_{n}, \omega_{n}\right)=\frac{\Delta^{k}\left[(\alpha[\beta+n])_{k-1} s_{n} / \omega_{n}\right]}{\Delta^{k}\left[(\alpha[\beta+n])_{k-1} / \omega_{n}\right]} \\
= & \frac{\sum_{j=0}^{k}(-1)^{j}\left(\begin{array}{l}
k \\
j
\end{array}\right) \frac{(\alpha[\beta+n+j])_{k-1}}{(\alpha[\beta+n+k])_{k-1}} \frac{s_{n+j}}{\omega_{n+j}}}{\sum_{j=0}^{k}(-1)^{j}\left(\begin{array}{l}
k \\
j
\end{array}\right) \frac{(\alpha[\beta+n+j])_{k-1}}{(\alpha[\beta+n+k])_{k-1}} \frac{1}{\omega_{n+j}}} \quad k, n \in \mathbb{N}_{0} .
\end{aligned}
$$

Depending upon the value of $\alpha>0$, the sequence transformation $\mathcal{C}_{k}^{(n)}\left(\alpha, \beta, s_{n}, \omega_{n}\right)$ interpolates between $\mathcal{S}_{k}^{(n)}\left(\beta, s_{n}, \omega_{n}\right)$ and Levin's sequence transformation $\mathcal{L}_{k}^{(n)}\left(\beta, s_{n}, \omega_{n}\right)$. If we choose $\alpha=1$ in (2.29) and compare the resulting expression with (2.21), we find

$$
\mathcal{C}_{k}^{(n)}\left(1, \beta, s_{n}, \omega_{n}\right)=\mathcal{S}_{k}^{(n)}\left(\beta, s_{n}, \omega_{n}\right)
$$


and if we use

$$
\lim _{\alpha \rightarrow \infty} \frac{(\alpha[\beta+n+j])_{k-1}}{\alpha^{k-1}}=\lim _{\alpha \rightarrow \infty} \prod_{m=1}^{k-1}(\beta+n+j+[m-1] / \alpha)=(\beta+n+j)^{k-1}
$$

in (2.29) and compare the resulting expression with (2.18), we find

$$
\lim _{\alpha \rightarrow \infty} \mathcal{C}_{k}^{(n)}\left(\alpha, \beta, s_{n}, \omega_{n}\right)=\mathcal{L}_{k}^{(n)}\left(\beta, s_{n}, \omega_{n}\right)
$$

Thus, the construction of explicit expressions for the Levin-type sequence transformations $\mathcal{G}_{k}^{(n)}\left(q_{m}, s_{n}, \omega_{n}\right), \mathcal{L}_{k}^{(n)}\left(\beta, s_{n}, \omega_{n}\right), \mathcal{S}_{k}^{(n)}\left(\beta, s_{n}, \omega_{n}\right), \mathcal{M}_{k}^{(n)}\left(\xi, s_{n}, \omega_{n}\right)$, and $\mathcal{C}_{k}^{(n)}\left(\alpha, \beta, s_{n}, \omega_{n}\right)$ with the help of annihilation operators is almost trivial. However, the annihilation operator approach does not only work in the case of the comparatively simple annihilating difference operators in (2.13), (2.17), (2.20), (2.24), and (2.28). In [132 Section 7.4] it was shown that Richardson extrapolation [104] and Sidi's generalized Richardson extrapolation process [123] can be derived by using divided differences as annihilation operators. Then, it was shown by Brezinski and Redivo Zaglia 32,33, Brezinski and Matos 30, and Matos 92 that the majority of the currently known transformations for scalar sequence can be derived via the annihilation operator approach. The generalization of this approach to vector and matrix sequences was discussed by Brezinski and Redivo Zaglia [34] and Brezinski and Salam [35].

\section{Recurrence Formulas}

In the theory of sequence transformations it is relatively uncommon that closed form expressions of the type of (1.1), 2.18), 2.21), 2.25), and (2.29) are known. The majority of the currently known sequence transformations are defined and computed via recursive schemes. From a computational point of view, the lack of an explicit expression is normally no disadvantage. The use of recurrence formulas is in most cases (much) much more efficient, in particular if a whole sequence of transforms must be computed simultaneously.

It is an additional advantage of the annihilation operator approach described in Section 2 that it permits a convenient construction of a recursive scheme for the numerators and denominators of $\mathcal{G}_{k}^{(n)}\left(q_{m}, s_{n}, \omega_{n}\right)$. For that purpose, let us define

$$
\begin{aligned}
& \Gamma_{k}^{(n)}=\Gamma_{k}^{(n)}\left(q_{m}, u_{n}\right)=\Delta^{k} X_{k}^{(n)}, \quad k, n \in \mathbb{N}_{0}, \\
& X_{k}^{(n)}=X_{k}^{(n)}\left(q_{m}, u_{n}\right)=\prod_{m=1}^{k-1}\left(n+q_{m}\right) u_{n}, \quad k, n \in \mathbb{N}_{0} .
\end{aligned}
$$

Comparison with (2.13) shows that $\Gamma_{k}^{(n)}$ corresponds apart from a missing phase factor $(-1)^{k}$ to the numerator in (2.15) if we choose $u_{n}=s_{n} / \omega_{n}$, and to the denominator in (2.15) if we choose $u_{n}=1 / \omega_{n}$.

The quantities $X_{k}^{(n)}$ satisfy for $k \geq 2$ the two-term recursion

$$
X_{k}^{(n)}=\left(n+q_{k-1}\right) X_{k-1}^{(n)} .
$$

Next, we use the commutator relationship (see [61] or [132 Eq. (7.2-2)])

$$
\Delta^{k}\left(n+q_{k-1}\right)-\left(n+q_{k-1}\right) \Delta^{k}=k E \Delta^{k-1},
$$

which can be proved by complete induction. This commutator can be rewritten as follows:

$$
\begin{aligned}
\Delta^{k}\left(n+q_{k-1}\right) & =\left(n+k+q_{k-1}\right) \Delta^{k}+k \Delta^{k-1} \\
& =\left[\left(n+k+q_{k-1}\right)(E-1)+k\right] \Delta^{k-1} \\
& =\left[\left(n+k+q_{k-1}\right) E-\left(n+q_{k-1}\right)\right] \Delta^{k-1} .
\end{aligned}
$$


Here, $E$ is the shift operator defined by $E f(n)=f(n+1)$.

Combination of (3.1), (3.2), (3.3) and (3.7) yields:

$$
\begin{aligned}
\Gamma_{k}^{(n)} & =\Delta^{k}\left(n+q_{k-1}\right) X_{k-1}^{(n)} \\
& =\left[\left(n+k+q_{k-1}\right) E-\left(n+q_{k-1}\right)\right] \Delta^{k-1} X_{k-1}^{(n)} \\
& =\left(n+k+q_{k-1}\right) \Gamma_{k-1}^{(n+1)}-\left(n+q_{k-1}\right) \Gamma_{k-1}^{(n)}, \quad k \geq 2, \quad n \in \mathbb{N}_{0} .
\end{aligned}
$$

Finally, we rescale $\Gamma_{k}^{(n)}$ according to

$$
G_{k}^{(n)}=G_{k}^{(n)}\left(q_{m}, u_{n}\right)=\frac{\Gamma_{k}^{(n)}\left(q_{m}, u_{n}\right)}{\prod_{m=1}^{k-1}\left(n+k+q_{m}\right)}, \quad k, n \in \mathbb{N}_{0}
$$

If we combine (3.10) and (3.11) and take into account that (3.1) and (3.2) imply $\Gamma_{0}^{(n)}=$ $u_{n}$ and $\Gamma_{1}^{(n)}=u_{n+1}-u_{n}$, respectively, we obtain the following recursive scheme for the numerators and denominators of the sequence transformation (1.1) introduced by Cížek, Zamastil, and Skála 48 Eq. (10)]:

$$
\begin{aligned}
G_{0}^{(n)} & =u_{n}, \quad n \in \mathbb{N}_{0}, \\
G_{1}^{(n)} & =u_{n+1}-u_{n}, \quad n \in \mathbb{N}_{0}, \\
G_{k+1}^{(n)} & =G_{k}^{(n+1)}-\frac{n+q_{k}}{n+k+q_{k}+1} \prod_{m=1}^{k-1} \frac{n+k+q_{m}}{n+k+q_{m}+1} G_{k}^{(n)}, \quad k \in \mathbb{N}, \quad n \in \mathbb{N}_{0} .
\end{aligned}
$$

If we choose $u_{n}=s_{n} / \omega_{n}$, this recursive scheme produces the numerator of (1.1), and if we choose $u_{n}=1 / \omega_{n}$, we obtain the denominator of (1.1).

As shown in [132 Sections 7.3, 8.3, and 9.3], recursive schemes for the numerator and denominator sums of the sequence transformations $\mathcal{L}_{k}^{(n)}\left(\beta, s_{n}, \omega_{n}\right) . \mathcal{S}_{k}^{(n)}\left(\beta, s_{n}, \omega_{n}\right)$, and $\mathcal{M}_{k}^{(n)}\left(\xi, s_{n}, \omega_{n}\right)$ can be derived in the same way. The recursive scheme [132, Eq. (7.2-8))]

$$
\begin{aligned}
L_{0}^{(n)} & =u_{n}, \quad n \in \mathbb{N}_{0}, \\
L_{k+1}^{(n)} & =L_{k}^{(n+1)}-\frac{(\beta+n)(\beta+n+k)^{k-1}}{(\beta+n+k+1)^{k}} L_{k}^{(n)}, \quad k, n \in \mathbb{N}_{0},
\end{aligned}
$$

produces the numerator and denominator sums in (2.18) if we choose $u_{n}=s_{n} / \omega_{n}$ and $u_{n}=1 / \omega_{n}$, respectively. We obtain (3.13) from (3.12) by choosing $q_{m}=\beta$.

Similarly, the recursive schemes [132, Eq. (8.3-7))]

$$
\begin{aligned}
S_{0}^{(n)} & =u_{n}, \quad n \in \mathbb{N}_{0}, \\
S_{k+1}^{(n)} & =S_{k}^{(n+1)}-\frac{(\beta+n+k-1)(\beta+n+k)}{(\beta+n+2 k-1)(\beta+n+2 k)} S_{k}^{(n)}, \quad k, n \in \mathbb{N}_{0},
\end{aligned}
$$

and [132 Eq. (9.3-6))]

$$
\begin{aligned}
& M_{0}^{(n)}=u_{n}, \quad n \in \mathbb{N}_{0}, \\
& M_{k+1}^{(n)}=M_{k}^{(n+1)}-\frac{\xi+n-k+1}{\xi+n+k+1} M_{k}^{(n)}, \quad k, n \in \mathbb{N}_{0},
\end{aligned}
$$

produce the numerator and denominator sums in (2.21) and (2.25), respectively. The recursive schemes (3.14) and (3.15) can be obtained from (3.12) by setting $q_{m}=\beta+m-1$ and $q_{m}=\xi-m+1$, respectively. 
If we set $q_{m}=\beta+[m-1] / \alpha$ in (3.12), we obtain the recursive scheme for the interpolating transformation $\mathcal{C}_{k}^{(n)}\left(\alpha, \beta, s_{n}, \omega_{n}\right)$ :

$$
\begin{aligned}
C_{0}^{(n)} & =u_{n}, \quad n \in \mathbb{N}_{0}, \\
C_{1}^{(n)} & =u_{n+1}-u_{n}, \quad n \in \mathbb{N}_{0} \\
C_{k+1}^{(n)} & =C_{k}^{(n+1)}-\frac{(\alpha[\beta+n]+k-1)(\alpha[\beta+n+k])_{k-1}}{(\alpha[\beta+n+k+1])_{k}} C_{k}^{(n)}, \quad k \in \mathbb{N}, n \in \mathbb{N}_{0} .
\end{aligned}
$$

This scheme produces the numerator and denominator sums of (2.29) if we choose $u_{n}=$ $s_{n} / \omega_{n}$ and $u_{n}=1 / \omega_{n}$, respectively. The recurrence formula published in [134. Eq. (3.3)] contains errors.

\section{Levin's Explicit Remainder Estimates}

It follows from (2.13), 2.14), (2.17), (2.20), (2.24), and (2.28) that all sequence transformations considered in this article can be expressed as follows:

$$
T_{k}^{(n)}\left(s_{n}, \omega_{n}\right)=\frac{\Delta^{k}\left\{P_{k-1}(n) s_{n} / \omega_{n}\right\}}{\Delta^{k}\left\{P_{k-1}(n) / \omega_{n}\right\}}, \quad k \in \mathbb{N}, \quad n \in \mathbb{N}_{0}
$$

Here, $P_{k-1}(n)$ is a polynomial of degree $k-1$ in $n$. Obviously, the remainder estimates $\left\{\omega_{n}\right\}_{n=0}^{\infty}$ have to satisfy the minimal requirement that $\Delta^{k}\left\{P_{k-1}(n) / \omega_{n}\right\} \neq 0$ for all finite $k, n \in \mathbb{N}_{0}$. In the following text, this will always be assumed.

The weighted difference operator $\Delta^{k} P_{k-1}(n)$ in (4.1) is linear. Accordingly, such a sequence transformation satisfies

$$
T_{k}^{(n)}\left(s_{n}, \omega_{n}\right)=s+\frac{\Delta^{k}\left\{P_{k-1}(n)\left[s_{n}-s\right] / \omega_{n}\right\}}{\Delta^{k}\left\{P_{k-1}(n) / \omega_{n}\right\}}, \quad k \in \mathbb{N}, \quad n \in \mathbb{N}_{0} .
$$

This property of Levin-type transformations has some far-reaching consequences. Let us assume that we can find for a given sequence $\left\{s_{n}\right\}_{n=0}^{\infty}$ a sequence $\left\{\omega_{n}\right\}_{n=0}^{\infty}$ of perfect remainder estimates such that

$$
s_{n}=s+r_{n}=s+c \omega_{n}, \quad c \neq 0, \quad n \in \mathbb{N}_{0} .
$$

The polynomial $P_{k-1}(n)$ in (4.1) is of degree $k-1$ in $n$, which implies that it is annihilated by $\Delta^{k}$ according to $\Delta^{k} P_{k-1}(n)=0$. Accordingly, the transformation problem is now trivial since (4.2) produces the (generalized) limit $s$ of the sequence (4.3) [132, Theorem 12-8]):

$$
T_{k}^{(n)}\left(s_{n}, \omega_{n}\right)=s+\frac{c \Delta^{k} P_{k-1}(n)}{\Delta^{k}\left\{P_{k-1}(n) / \omega_{n}\right\}}=s, \quad k \in \mathbb{N}, \quad n \in \mathbb{N}_{0} .
$$

Unfortunately, perfect remainder estimates satisfying (4.3) can only be found for practically more or less irrelevant model problems. In the case of realistic problems, we have to be more modest and can only hope to find remainder estimates that reproduce the leading order asymptotics of the actual remainders [132] Eq. (7.3-1)]:

$$
r_{n}=s_{n}-s=\omega_{n}[c+\mathrm{O}(1 / n)], \quad c \neq 0, \quad n \rightarrow \infty .
$$

This asymptotic condition does not fix remainder estimates uniquely. All Levin-type transformations considered in this article are invariant under the transformation $\omega_{n} \rightarrow c \omega_{n}$ with 
$c \neq 0$. Moreover, given a sequence $\left\{r_{n}\right\}_{n=0}^{\infty}$ of remainders it is usually possible to find a variety of genuinely different sequences $\left\{\omega_{n}\right\}_{n=0}^{\infty},\left\{\omega_{n}^{\prime}\right\}_{n=0}^{\infty},\left\{\omega_{n}^{\prime \prime}\right\}_{n=0}^{\infty}, \ldots$ of remainder estimates which all satisfy the asymptotic condition 4.5.

In some exceptional cases, explicit analytical expressions for remainder estimates can be found. Let us for instance assume that the elements of the sequence to be transformed are the partial sums $s_{n}=\sum_{k=0}^{n} a_{k}$ of an infinite series and that the terms $a_{k}$ have a sufficiently simple analytical structure. Then it may be possible to derive an explicit expression for the truncation error, from which explicit remainder estimates satisfying (4.5) can be derived.

In principle, such an analytical approach would be highly desirable, in particular since it should then be possible to construct for a given sequence $\left\{s_{n}\right\}_{n=0}^{\infty}$ more effective remainder estimates $\left\{\tilde{\omega}_{n}^{(l)}\right\}_{n=0}^{\infty}$ that do not only reproduce the leading order asymptotics of the remainders according to (4.5), but several of the leading orders according to

$$
r_{n}=s_{n}-s=\tilde{\omega}_{n}^{(l)}\left[c+\mathrm{O}\left(n^{-l}\right)\right], \quad c \neq 0, \quad n \rightarrow \infty,
$$

where $l>1$ is a fixed positive integer. Improved remainder estimates of that kind should lead to more efficient Levin-type transformations. Unfortunately, only relatively little work has been done on the asymptotics of truncation errors $\sum_{k=n+1}^{\infty} a_{k}$ of infinite series as $n \rightarrow \infty$ beyond the leading order. Moreover, in many applications of Levin-type transformations in particular in physics, only the numerical values of a finite string of sequence elements or series coefficients are available, but no explicit analytical expressions. In such a case, remainder estimates have to be constructed from the numerical values of the input data via simple rules.

Levin-type sequence transformations are not limited to strongly divergent perturbation expansions. They are able to accelerate the convergence of many series and sequences if suitable remainder estimates are used. In this context, it is helpful to introduce first some terminology which is common in the literature on convergence acceleration methods. Many practically relevant sequences $\left\{s_{n}\right\}_{n=0}^{\infty}$, which converge to some limit $s$, satisfy

$$
\lim _{n \rightarrow \infty} \frac{s_{n+1}-s}{s_{n}-s}=\lim _{n \rightarrow \infty} \frac{r_{n+1}}{r_{n}}=\rho .
$$

If $0<|\rho|<1$ holds, we say that the sequence $\left\{s_{n}\right\}_{n=0}^{\infty}$ converges linearly, if $\rho=1$ holds, we say that this sequence converges logarithmically, and if $\rho=0$ holds, we say that it converges hyperlinearly. Of course, $|\rho|>1$ implies that the sequence $\left\{s_{n}\right\}_{n=0}^{\infty}$ diverges.

On the basis of purely heuristic arguments Levin [90 and later Smith and Ford [126] suggested some simple remainder estimates which according to experience nevertheless work remarkably well in a large variety of cases. These simple remainder estimates can be motivated by considering simple model problems. For that purpose, let us assume that the elements of a sequence $\left\{s_{n}\right\}_{n=0}^{\infty}$ of partial sums $\sum_{\nu=0}^{n} a_{\nu}$ behave as follows:

$$
s_{n} \sim s+z^{n+1} n^{\theta}\left[\alpha_{0}+\frac{\alpha_{1}}{n}+\frac{\alpha_{2}}{n^{2}}+\ldots\right], \quad n \rightarrow \infty .
$$

This is a fairly general model sequence, which is able to describe the asymptotics of many practically relevant sequences as $n \rightarrow \infty$ and which Levin [90 had in mind when he introduced his simple remainder estimates. For $|z|<1$, the sequence (4.8) converges linearly to its limit $s$, for $z=1$ and $\operatorname{Re}(\theta)<0$, it converges logarithmically, and for $|z|>1$ it diverges.

From (4.8) we obtain via $a_{n}=\Delta s_{n-1}$ the leading orders of the asymptotic expansion of the terms of the infinite series $\sum_{\nu=0}^{\infty} a_{\nu}$. For $z=1$ and $\operatorname{Re}(\theta)<0$ (logarithmic convergence), we find with the help of the computer algebra system Maple

$$
\begin{aligned}
a_{n}=n^{\theta}\left\{\frac{\theta \alpha_{0}}{n}+\frac{(\theta-1)\left[2 \alpha_{1}-\theta \alpha_{0}\right]}{2 n^{2}}\right. \\
\left.+\frac{(\theta-2)\left[6 \alpha_{2}+(\theta-1)\left\{\theta \alpha_{0}-3 \alpha_{1}\right\}\right]}{6 n^{3}}+\mathrm{O}\left(n^{-3}\right)\right\}, \quad n \rightarrow \infty,
\end{aligned}
$$


and for $|z|<1$ (linear convergence), we find.

$$
\begin{aligned}
a_{n}=z^{n} n^{\theta}\left\{(z-1) \alpha_{0}+\frac{\theta \alpha_{0}+(z-1) \alpha_{1}}{n}\right. & \\
+ & \left.\frac{(\theta-1)\left[2 \alpha_{1}-\theta \alpha_{0}\right]+2(z-1) \alpha_{2}}{2 n^{2}}+\mathrm{O}\left(n^{-3}\right)\right\}, \quad n \rightarrow \infty .
\end{aligned}
$$

If we compare (4.8) and (4.9), we see that in the case of logarithmic convergence the term $a_{n}=\Delta s_{n-1}=\mathrm{O}\left(n^{\theta-1}\right)$ cannot reproduce the leading order of the remainder $r_{n}=$ $s_{n}-s=\mathrm{O}\left(n^{\theta}\right)$. However, the product $n a_{n}=\mathrm{O}\left(n^{\theta}\right)$ reproduces the leading order of the remainder of the model sequence (4.8). Thus, it is an obvious idea to use the remainder estimate 90 .

$$
\omega_{n}=(\beta+n) \Delta s_{n-1}=(\beta+n) a_{n}
$$

in Levin's general transformation (2.18), yielding Levin's $u$ transformation in the notation of [132, Eq. (7.3-5)]:

$$
u_{k}^{(n)}\left(\beta, s_{n}\right)=\mathcal{L}_{k}^{(n)}\left(\beta, s_{n},(\beta+n) \Delta s_{n-1}\right), \quad k, n \in \mathbb{N}_{0} .
$$

Since Levin's remainder estimate (4.11) reproduces the leading order of the remainder of the model sequence (4.8) for $z=1$ and $\operatorname{Re}(\theta)<0$ (logarithmic convergence), it is not surprising that the $u$ transformation is an effective accelerator for many monotone, logarithmically convergent sequences and series.

In the case of linear convergence $(|z|<1$ in (4.8), the asymptotic expansion (4.10) indicates that the term $a_{n}$ itself and not the product $n a_{n}$ would be a natural estimate for the truncation error in (4.8). However, the Levin-type transformations considered in this article nevertheless accelerate convergence if instead of the "right" sequence $\left\{\omega_{n}\right\}_{n=0}^{\infty}$ of remainder estimates "wrong" remainder estimates $\omega_{n}^{\prime}=(n+\beta)^{l} \omega_{n}$ with $l \in \mathbb{N}_{0}$ are used (see Theorems 12-14 - 12-16 and the discussion on pp. 310 - 311 of [132]). The use of "wrong" remainder estimates only leads to a decrease of the efficiency of the transformation process (compare for instance [132 Theorem 13-12]), depending on the magnitude of $l$. With the help of a generalization of Germain-Bonne's formal theory of convergence acceleration 64 it can be proved rigorously that the $u$ transformation accelerates linear convergence [132] Theorems $12-10,12-11$, and 12-16].

Moreover, the $u$ transformation is also capable of summing effectively many alternating divergent series. According to Smith and Ford [126, 127] the $u$ transformation is among the most versatile and powerful sequence transformations that are currently known. This explains why Levin's $u$ transformation is used internally in the computer algebra system Maple in the case of convergence problems (see for example [49, pp. 51 and 125] or [71, p. $258]$ ).

The remainder estimate (4.11) can also be inserted into the explicit expressions (2.21), (2.25), and (2.29) for $\mathcal{S}_{k}^{(n)}\left(\beta, s_{n}, \omega_{n}\right), \mathcal{M}_{k}^{(n)}\left(\xi, s_{n}, \omega_{n}\right)$, and $\mathcal{C}_{k}^{(n)}\left(\alpha, \beta, s_{n}, \omega_{n}\right)$, yielding the $u$-type variants [132, Eqs. (8.4-2) and (9.4-2)]

$$
\begin{aligned}
y_{k}^{(n)}\left(\beta, s_{n}\right) & =\mathcal{S}_{k}^{(n)}\left(\beta, s_{n},(\beta+n) \Delta s_{n-1}\right), \quad k, n \in \mathbb{N}_{0}, \\
Y_{k}^{(n)}\left(\xi, s_{n}\right) & =\mathcal{M}_{k}^{(n)}\left(\xi, s_{n},(-\xi-n) \Delta s_{n-1}\right), \quad k, n \in \mathbb{N}_{0}, \\
{ }_{u} \mathcal{C}_{k}^{(n)}\left(\alpha, \beta, s_{n}\right) & =\mathcal{C}_{k}^{(n)}\left(\alpha, \beta, s_{n},(\beta+n) \Delta s_{n-1}\right), \quad k, n \in \mathbb{N}_{0} .
\end{aligned}
$$

In the case of the sequence transformation $\mathcal{G}_{k}^{(n)}\left(q_{m}, s_{n}, \omega_{n}\right)$ introduced by Č́ížek, Zamastil, and Skála [48. Eq. (10)] we choose the $u$-type remainder estimates according to

$$
\omega_{n}=\left(n+q_{0}\right) \Delta s_{n-1}=\left(n+q_{0}\right) a_{n},
$$


where $q_{0} \geq 0$ is a suitable constant. Inserting this into (1.1) yields:

$$
\begin{aligned}
& { }_{u} \mathcal{G}_{k}^{(n)}\left(q_{m}, s_{n}\right)=\mathcal{G}_{k}^{(n)}\left(q_{m}, s_{n},\left(n+q_{0}\right) \Delta s_{n-1}\right) \\
& =\frac{\sum_{j=0}^{k}(-1)^{j}\left(\begin{array}{l}
k \\
j
\end{array}\right) \prod_{m=1}^{k-1} \frac{\left(n+j+q_{m}\right)}{\left(n+k+q_{m}\right)} \frac{s_{n+j}}{\left(n+j+q_{0}\right) \Delta s_{n+j-1}}}{\sum_{j=0}^{k}(-1)^{j}\left(\begin{array}{l}
k \\
j
\end{array}\right) \prod_{m=1}^{k-1} \frac{\left(n+j+q_{m}\right)}{\left(n+k+q_{m}\right)} \frac{1}{\left(n+j+q_{0}\right) \Delta s_{n+j-1}}}, \quad k, n \in \mathbb{N}_{0} .
\end{aligned}
$$

As discussed in more details in Section 6] (see the discussion following (6.13)), this $u$-type transformation looses some important exactness properties if $q_{0}>0$ is essentially arbitrary and does not satisfy $q_{0} \in\left\{q_{1}, \ldots, q_{m}\right\}$ because then $\left[\prod_{m=1}^{k-1}\left(n+q_{m}\right)\right] /\left(n+q_{0}\right)$ is rational in $n$ and does not simplify to a polynomial of degree $k-2$ in $n$. Thus, an obvious idea would be to choose $q_{0}=q_{1}$. The other $u$-type transformations (4.12), (4.13), (4.14), and (4.15) satisfy this requirement.

The asymptotic expansion (4.10) indicates that in the case linear convergence the term $a_{n}$ is a natural estimate for the truncation error of the sequence (4.8) with $|z|<1$. Thus, Levin 90 proposed for linearly convergent sequences and series the remainder estimate

$$
\omega_{n}=\Delta s_{n-1}=a_{n}
$$

which yields Levin's $t$ transformation in the notation of [132, Eq. (7.3-7)]:

$$
t_{k}^{(n)}\left(\beta, s_{n}\right)=\mathcal{L}_{k}^{(n)}\left(\beta, s_{n}, \Delta s_{n-1}\right), \quad k, n \in \mathbb{N}_{0} .
$$

The $t$ transformation is an effective accelerator for linear convergence and in particular for alternating series [126, 127. With the help of a generalization of Germain-Bonne's formal theory of convergence acceleration 64 it can be proved rigorously that Levin's $t$ transformation accelerates linear convergence [132 Theorems 12-10, 12-11, and 12-16]. It is also able to sum many alternating divergent series. However, a comparison of (4.8) and (4.9) indicates that the $t$ transformation should fail to accelerate logarithmic convergence (for more details, see [132 Theorem 14-1]).

The use of the remainder estimate (4.18) in the explicit expressions (2.21), (2.25), and (2.29) for $\mathcal{S}_{k}^{(n)}\left(\beta, s_{n}, \omega_{n}\right), \mathcal{M}_{k}^{(n)}\left(\xi, s_{n}, \omega_{n}\right)$, and $\mathcal{C}_{k}^{(n)}\left(\alpha, \beta, s_{n}, \omega_{n}\right)$ yields the $t$-type variants 132 Eqs. (8.4-3) and (9.4-3)]

$$
\begin{array}{rlrl}
\tau_{k}^{(n)}\left(\beta, s_{n}\right) & =\mathcal{S}_{k}^{(n)}\left(\beta, s_{n}, \Delta s_{n-1}\right), \quad k, n \in \mathbb{N}_{0}, \\
T_{k}^{(n)}\left(\xi, s_{n}\right) & =\mathcal{M}_{k}^{(n)}\left(\xi, s_{n}, \Delta s_{n-1}\right), & k, n \in \mathbb{N}_{0}, \\
{ }_{t} \mathcal{C}_{k}^{(n)}\left(\alpha, \beta, s_{n}\right) & =\mathcal{C}_{k}^{(n)}\left(\alpha, \beta, s_{n}, \Delta s_{n-1}\right), \quad k, n \in \mathbb{N}_{0} .
\end{array}
$$

In the case of the sequence transformation $\mathcal{G}_{k}^{(n)}\left(q_{m}, s_{n}, \omega_{n}\right)$ we obtain in this way:

$$
\begin{aligned}
{ }_{t} \mathcal{G}_{k}^{(n)}\left(q_{m}, s_{n}\right)= & \mathcal{G}_{k}^{(n)}\left(q_{m}, s_{n}, \Delta s_{n-1}\right) \\
= & \frac{\sum_{j=0}^{k}(-1)^{j}\left(\begin{array}{l}
k \\
j
\end{array}\right) \prod_{m=1}^{k-1} \frac{\left(n+j+q_{m}\right)}{\left(n+k+q_{m}\right)} \frac{s_{n+j}}{\Delta s_{n+j-1}}}{\sum_{j=0}^{k}(-1)^{j}\left(\begin{array}{l}
k \\
j
\end{array}\right) \prod_{m=1}^{k-1} \frac{\left(n+j+q_{m}\right)}{\left(n+k+q_{m}\right)} \frac{1}{\Delta s_{n+j-1}}}, \quad k, n \in \mathbb{N}_{0} .
\end{aligned}
$$

Inspired by Aitken's $\Delta^{2}$ formula [1, Levin 90] introduced as a third simple remainder estimate

$$
\omega_{n}=\frac{\Delta s_{n-1} \Delta s_{n}}{\Delta s_{n-1}-\Delta s_{n}}=\frac{a_{n} a_{n+1}}{a_{n}-a_{n+1}} .
$$


The usefulness of this remainder estimate can be demonstrated by applying it to the model sequence (4.8). For $\zeta=1$ and $\operatorname{Re}(\theta)<0$ (logarithmic convergence) we obtain

$$
\begin{aligned}
& \frac{a_{n} a_{n+1}}{a_{n}-a_{n+1}}=n^{\theta}\left\{-\frac{\theta \alpha_{0}}{\theta-1}-\frac{\theta \alpha_{1}}{(\theta-1) n}\right. \\
& \left.\quad+\left[(\theta+1)\left\{\frac{\theta \alpha_{0}}{12}-\frac{(\theta-2) \alpha_{2}}{\theta-1}\right\}-\frac{\alpha_{1}^{2}}{\theta \alpha_{0}}\right] \frac{1}{(\theta-1) n^{2}}+\mathrm{O}\left(n^{-3}\right)\right\}, \quad n \rightarrow \infty,
\end{aligned}
$$

and for $|z|<1$ (linear convergence) we obtain

$$
\frac{a_{n} a_{n+1}}{a_{n}-a_{n+1}}=z^{n+1} n^{\theta}\left\{-\alpha_{0}-\frac{\alpha_{1}}{n}-\left[\frac{z \theta \alpha_{0}}{(z-1)^{2}}+\alpha_{2}\right] \frac{1}{n^{2}}+\mathrm{O}\left(n^{-3}\right)\right\}, \quad n \rightarrow \infty .
$$

It is a remarkable feature of the remainder estimate (4.24) that it does not only reproduce the leading order of the model sequence (4.8), but both in the case of linear and logarithmic convergence also the next one. Thus, the reminder estimate (4.24) is in the case of the model sequence (4.8) a remainder estimate of the type of (4.6) with $l=2$, whereas Levin's other two remainder estimates (4.11) and (4.18) only satisfy (4.5). Of course, it would be desirable to find other remainder estimate which are also able to reproduce more than the leading order asymptotics of the truncation error. Further research into this direction should be of considerable interest.

The use of the remainder estimate (4.24) in (2.18) yields Levin's $v$ transformation in the notation of 132 Eq. (7.3-11)]:

$$
v_{k}^{(n)}\left(\beta, s_{n}\right)=\mathcal{L}_{k}^{(n)}\left(\beta, s_{n}, \Delta s_{n-1} \Delta s_{n} /\left[\Delta s_{n-1}-\Delta s_{n}\right]\right), \quad k, n \in \mathbb{N}_{0} .
$$

Levin's $v$ transformation is an effective accelerator for many linearly and logarithmically convergent sequences and series. With the help of a generalization of Germain-Bonne's formal theory of convergence acceleration 64] it can be proved rigorously that the $v$ transformation accelerates linear convergence [132, Theorems 12-10 and 12-11]. The $v$ transformation is also able to sum many alternating divergent series. According to Smith and Ford 126, 127, the $v$ transformation has similar properties as the $u$ transformation, which means that it is among the most versatile and powerful sequence transformations that are currently known.

The use of the remainder estimate (4.24) in the explicit expressions (2.21), (2.25), and (2.29) for $\mathcal{S}_{k}^{(n)}\left(\beta, s_{n}, \omega_{n}\right), \mathcal{M}_{k}^{(n)}\left(\xi, s_{n}, \omega_{n}\right)$, and $\mathcal{C}_{k}^{(n)}\left(\alpha, \beta, s_{n}, \omega_{n}\right)$ yields the $v$-type variants 132 Eqs. (8.4-5) and (9.4-5)]

$$
\begin{aligned}
\varphi_{k}^{(n)}\left(\beta, s_{n}\right) & =\mathcal{S}_{k}^{(n)}\left(\beta, s_{n}, \Delta s_{n-1} \Delta s_{n} /\left[\Delta s_{n-1}-\Delta s_{n}\right]\right), \quad k, n \in \mathbb{N}_{0}, \\
\Phi_{k}^{(n)}\left(\xi, s_{n}\right) & =\mathcal{M}_{k}^{(n)}\left(\xi, s_{n}, \Delta s_{n-1} \Delta s_{n} /\left[\Delta s_{n-1}-\Delta s_{n}\right]\right), \quad k, n \in \mathbb{N}_{0} . \\
{ }_{t} \mathcal{C}_{k}^{(n)}\left(\alpha, \beta, s_{n}\right) & =\mathcal{C}_{k}^{(n)}\left(\alpha, \beta, s_{n}, \Delta s_{n-1} \Delta s_{n} /\left[\Delta s_{n-1}-\Delta s_{n}\right]\right), \quad k, n \in \mathbb{N}_{0} .
\end{aligned}
$$

In the case of the sequence transformation $\mathcal{G}_{k}^{(n)}\left(q_{m}, s_{n}, \omega_{n}\right)$ we obtain

$$
\begin{aligned}
{ }_{v} \mathcal{G}_{k}^{(n)}\left(q_{m}, s_{n}\right)=\mathcal{G}_{k}^{(n)}\left(q_{m}, s_{n}, \Delta s_{n-1} \Delta s_{n} /\left[\Delta s_{n-1}-\Delta s_{n}\right]\right) \\
=\frac{\sum_{j=0}^{k}(-1)^{j}\left(\begin{array}{c}
k \\
j
\end{array}\right) \prod_{m=1}^{k-1} \frac{\left(n+j+q_{m}\right)}{\left(n+k+q_{m}\right)} \frac{\left(\Delta s_{n+j-1}-\Delta s_{n+j}\right) s_{n+j}}{\Delta s_{n+j-1} \Delta s_{n+j}}}{\sum_{j=0}^{k}(-1)^{j}\left(\begin{array}{c}
k \\
j
\end{array}\right) \prod_{m=1}^{k-1} \frac{\left(n+j+q_{m}\right)}{\left(n+k+q_{m}\right)} \frac{\Delta s_{n+j-1}-\Delta s_{n+j}}{\Delta s_{n+j-1} \Delta s_{n+j}}}, \quad k, n \in \mathbb{N}_{0} .
\end{aligned}
$$


The best simple estimate for the truncation error of a strictly alternating convergent series is the first term not included in the partial sum [88, p. 259]. Moreover, the first term neglected is also an estimate of the truncation error of a divergent hypergeometric series ${ }_{2} F_{0}(a, b,-z)$ with $a, b, z>0$ [38, Theorem 5.12-5]. Accordingly, Smith and Ford [126] proposed the remainder estimate

$$
\omega_{n}=\Delta s_{n}=a_{n}
$$

This remainder estimate can also be motivated via the model sequence (4.8). For $z=1$ and $\operatorname{Re}(\theta)<0$ (logarithmic convergence), we find

$$
\begin{aligned}
a_{n+1}=n^{\theta} & \left\{\frac{\theta \alpha_{0}}{n}+\frac{(\theta-1)\left[2 \alpha_{1}+\theta \alpha_{0}\right]}{2 n^{2}}\right. \\
& \left.+\frac{(\theta-2)\left[6 \alpha_{2}+(\theta-1)\left\{\theta \alpha_{0}+3 \alpha_{1}\right\}\right]}{6 n^{3}}+\mathrm{O}\left(n^{-3}\right)\right\}, \quad n \rightarrow \infty,
\end{aligned}
$$

and for $|z|<1$ (linear convergence), we find.

$$
\begin{aligned}
a_{n+1}=z^{n+1} & n^{\theta}\left\{(z-1) \alpha_{0}+\frac{z \theta \alpha_{0}+(z-1) \alpha_{1}}{n}\right. \\
+ & \left.\frac{z(\theta-1)\left[2 \alpha_{1}+\theta \alpha_{0}\right]+2(z-1) \alpha_{2}}{2 n^{2}}+\mathrm{O}\left(n^{-3}\right)\right\}, \quad n \rightarrow \infty .
\end{aligned}
$$

The use of the remainder estimate (4.32) yields Levin's $d$ transformation in the notation of 132 Eq. (7.3-9)]:

$$
d_{k}^{(n)}\left(\beta, s_{n}\right)=\mathcal{L}_{k}^{(n)}\left(\beta, s_{n}, \Delta s_{n}\right), \quad k, n \in \mathbb{N}_{0}
$$

If we compare (4.33) with (4.9) and (4.34) with (4.10), we see that Levin's $d$ transformation should have similar properties as Levin's $t$ transformation. This is confirmed by experience: The $d$ transformation is a powerful accelerator for linear convergence and in particular for alternating series, and is also able to sum many alternating divergent series, but fails to accelerate logarithmic convergence. With the help of a generalization of Germain-Bonne's formal theory of convergence acceleration [64 it can be proved rigorously that the $d$ transformation accelerates linear convergence 132 Theorems 12-10, 12-11, and 12-16].

The use of the remainder estimate (4.32) in the explicit expressions (2.21), (2.25), and (2.29) for $\mathcal{S}_{k}^{(n)}\left(\beta, s_{n}, \omega_{n}\right), \mathcal{M}_{k}^{(n)}\left(\xi, s_{n}, \omega_{n}\right)$, and $\mathcal{C}_{k}^{(n)}\left(\alpha, \beta, s_{n}, \omega_{n}\right)$ yields the $d$-type variants 132 Eqs. (8.4-4) and (9.4-4)]

$$
\begin{aligned}
\delta_{k}^{(n)}\left(\beta, s_{n}\right) & =\mathcal{S}_{k}^{(n)}\left(\beta, s_{n}, \Delta s_{n}\right), \quad k, n \in \mathbb{N}_{0}, \\
\Delta_{k}^{(n)}\left(\xi, s_{n}\right) & =\mathcal{M}_{k}^{(n)}\left(\xi, s_{n}, \Delta s_{n}\right), \quad k, n \in \mathbb{N}_{0}, \\
{ }_{d} \mathcal{C}_{k}^{(n)}\left(\alpha, \beta, s_{n}\right) & =\mathcal{C}_{k}^{(n)}\left(\alpha, \beta, s_{n}, \Delta s_{n}\right), \quad k, n \in \mathbb{N}_{0} .
\end{aligned}
$$

As already mentioned in Section 1 the delta transformation (4.36) was found to be particularly powerful in the case of factorially and hyperfactorially divergent alternating power series as they for instance occur in the perturbation expansions of quantum physics $174647487783132133134,135136137,138139141142144148149$ or in asymptotic expansions for special functions [133, 136, 140, 147]). 
In the case of the sequence transformation $\mathcal{G}_{k}^{(n)}\left(q_{m}, s_{n}, \omega_{n}\right)$ we obtain

$$
\begin{aligned}
{ }_{d} \mathcal{G}_{k}^{(n)}\left(q_{m}, s_{n}\right)= & \mathcal{G}_{k}^{(n)}\left(q_{m}, s_{n}, \Delta s_{n}\right) \\
= & \frac{\sum_{j=0}^{k}(-1)^{j}\left(\begin{array}{l}
k \\
j
\end{array}\right) \prod_{m=1}^{k-1} \frac{\left(n+j+q_{m}\right)}{\left(n+k+q_{m}\right)} \frac{s_{n+j}}{\Delta s_{n+j}}}{\sum_{j=0}^{k}(-1)^{j}\left(\begin{array}{l}
k \\
j
\end{array}\right) \prod_{m=1}^{k-1} \frac{\left(n+j+q_{m}\right)}{\left(n+k+q_{m}\right)} \frac{1}{\Delta s_{n+j}}}, \quad k, n \in \mathbb{N}_{0} .
\end{aligned}
$$

In practical applications it happens relatively often that asymptotic expressions $a_{n}^{(\infty)}$ for the terms $a_{n}$ of a series are known that reproduce the leading order of $a_{n}$ as $n \rightarrow \infty$. For example, the coefficients $c_{n}$ of many divergent perturbation expansions, which are power series in some coupling constant $g$, satisfy (see for example [62 Table 1])

$$
c_{n}=(-1)^{n} \Gamma(a n+b) R^{n}[C+\mathrm{O}(1 / n)], \quad n \rightarrow \infty,
$$

where $a, b, C$, and $R$ are known constants.

The remainder estimate (4.24), which leads to Levin's $v$ transformation, is in some sense exceptional since reproduces not only the leading order of the truncation errors of the model sequence (4.8) as $n \rightarrow \infty$, but also the next one. Normally, we can only expect that the simple remainder estimates (4.11), 4.18), 4.24), and (4.32) reproduce the leading order of the remainder $s_{n}-s$. However, the leading order of the truncation error is also reproduced if we use in (4.11), (4.18), (4.24), and (4.32) not $a_{n}$ and $a_{n+1}$ but their limiting expressions $a_{n}^{(\infty)}$ and $a_{n+1}^{(\infty)}$. Whether this improves the transformation results or not, depends on the problem under consideration and cannot be decided by simple considerations. Nevertheless, it may well be worth a try. Ideas of that kind were discussed in more details in 75 [35] and also by Čížek, Zamastil, and Skála 48.

The $t$-type transformations (4.19), (4.20), (4.21), (4.22), and (4.23) use the last term $a_{n}$ of the partial sum $s_{n}=\sum_{k=0}^{n} a_{k}$ as an estimate for the truncation error $r_{n}=-\sum_{k=n+1}^{\infty} a_{k}$, whereas the analogous $d$-type transformations (4.35), (4.36), (4.37), (4.38), and (4.39) use the first term $a_{n+1}$ not included in the partial sum as the remainder estimate. Thus, it looks that $t$-type transformation utilize the available information in some sense more effectively than $d$-type transformation since they use $a_{n+1}$ also for the construction of the next partial sum $s_{n+1}$. This is, however, a superficial judgment and it can be shown easily that $t$-type transformations are actually $d$-type transformations is disguise.

All $t$-type transformations of this article can be expressed as follows:

$$
\begin{aligned}
{ }_{t} T_{k}^{(n)}\left(s_{n}, \Delta s_{n-1}\right)= & \frac{\Delta^{k}\left[P_{k-1}(n) s_{n} / \Delta s_{n-1}\right]}{\Delta^{k}\left[P_{k-1}(n) / \Delta s_{n-1}\right]} \\
& =\frac{\sum_{j=0}^{k}(-1)^{j}\left(\begin{array}{l}
k \\
j
\end{array}\right) P_{k-1}(n+j) \frac{s_{n+j}}{\Delta s_{n+j-1}}}{\sum_{j=0}^{k}(-1)^{j}\left(\begin{array}{l}
k \\
j
\end{array}\right) P_{k-1}(n+j) \frac{1}{\Delta s_{n+j-1}}}, \quad k \in \mathbb{N}, \quad n \in \mathbb{N}_{0} .
\end{aligned}
$$

As usual, $P_{k-1}(n)$ is a polynomial of degree $k-1$ in $n$.

Let us now use the relationship $s_{n}=s_{n-1}+\Delta s_{n-1}$ in the numerator on the right-hand side of (4.41). In view of the linearity of $\Delta^{k}$ we then obtain:

$$
\Delta^{k} \frac{P_{k-1}(n) s_{n}}{\Delta s_{n-1}}=\Delta^{k}\left\{P_{k-1}(n)\left[\frac{s_{n-1}}{\Delta s_{n-1}}+\frac{\Delta s_{n-1}}{\Delta s_{n-1}}\right]\right\}=\Delta^{k} \frac{P_{k-1}(n) s_{n-1}}{\Delta s_{n-1}}
$$


Inserting this into (4.41) and (4.42) shows that a $t$-type transformation is actually a $d$-type transformation with a transformed polynomial $\mathcal{P}_{k-1}(n-1)=P_{k-1}(n)$ :

$$
\begin{aligned}
{ }_{t} T_{k}^{(n)}\left(s_{n}, \Delta s_{n-1}\right)= & \frac{\Delta^{k}\left[\mathcal{P}_{k-1}(n-1) s_{n-1} / \Delta s_{n-1}\right]}{\Delta^{k}\left[\mathcal{P}_{k-1}(n-1) / \Delta s_{n-1}\right]} \\
& =\frac{\sum_{j=0}^{k}(-1)^{j}\left(\begin{array}{c}
k \\
j
\end{array}\right) \mathcal{P}_{k-1}(n+j-1) \frac{s_{n+j-1}}{\Delta s_{n+j-1}}}{\sum_{j=0}^{k}(-1)^{j}\left(\begin{array}{c}
k \\
j
\end{array}\right) \mathcal{P}_{k-1}(n+j-1) \frac{1}{\Delta s_{n+j-1}}} .
\end{aligned}
$$

Thus, the $t$-type transformations defined in (4.19), (4.20), (4.21), (4.22), and (4.23) can be expressed by the corresponding $d$-type transformations defined in (4.35), (4.36), 4.37), (4.38), and (4.39) according to

$$
\begin{aligned}
t_{k}^{(n)}\left(\beta, s_{n}\right) & =\mathcal{L}_{k}^{(n-1)}\left(\beta+1, s_{n-1}, \Delta s_{n-1}\right)=d_{k}^{(n-1)}\left(\beta+1, s_{n-1}\right), \\
\tau_{k}^{(n)}\left(\beta, s_{n}\right) & =\mathcal{S}_{k}^{(n-1)}\left(\beta+1, s_{n-1}, \Delta s_{n-1}\right)=\delta_{k}^{(n-1)}\left(\beta+1, s_{n-1}\right), \\
T_{k}^{(n)}\left(\xi, s_{n}\right) & =\mathcal{M}_{k}^{(n-1)}\left(\xi+1, s_{n-1}, \Delta s_{n-1}\right)=\Delta_{k}^{(n-1)}\left(\xi+1, s_{n-1}\right), \\
{ }_{t} \mathcal{C}_{k}^{(n)}\left(\alpha, \beta, s_{n}\right) & =\mathcal{C}_{k}^{(n-1)}\left(\alpha, \beta+1, s_{n-1}, \Delta s_{n-1}\right)={ }_{d} \mathcal{C}_{k}^{(n)}\left(\alpha, \beta+1, s_{n-1}\right), \\
{ }_{t} \mathcal{G}_{k}^{(n)}\left(q_{m}, s_{n}\right) & =\mathcal{G}_{k}^{(n-1)}\left(q_{m}+1, s_{n-1}, \Delta s_{n-1}={ }_{d} \mathcal{G}_{k}^{(n-1)}\left(q_{m}+1, s_{n-1}\right) .\right.
\end{aligned}
$$

In these expressions, the case $n=0$ deserves special consideration. Since we tacitly assume $s_{-m}=0$ with $m \in \mathbb{N}$, the term with $j=0$ in the numerator sum of (4.45) vanishes for $n=0$. This can also be proved directly from the numerator sum in (4.42). If we write there $\Delta s_{n+j-1}=a_{n+j}$ and $s_{n+j}=\sum_{\nu=0}^{n+j} a_{\nu}$, we obtain for $n=0$ :

$$
\begin{aligned}
\sum_{j=0}^{k}(-1)^{j}\left(\begin{array}{l}
k \\
j
\end{array}\right) P_{k-1}(j) \sum_{\nu=0}^{j} \frac{a_{\nu}}{a_{j}} & =\sum_{j=0}^{k}(-1)^{j}\left(\begin{array}{l}
k \\
j
\end{array}\right) P_{k-1}(j)\left[\sum_{\nu=0}^{j-1} \frac{a_{\nu}}{a_{j}}+\frac{a_{j}}{a_{j}}\right] \\
& =\sum_{j=1}^{k}(-1)^{j}\left(\begin{array}{l}
k \\
j
\end{array}\right) P_{k-1}(j) \sum_{\nu=0}^{j-1} \frac{a_{\nu}}{a_{j}}
\end{aligned}
$$

Here, we made use of the fact that $\sum_{\nu=0}^{j-1} a_{\nu}$ is for $j=0$ an empty sum which is zero. Thus, for $n=0$ the general $t$-type transformation (4.42) can be expressed as follows:

$$
\begin{aligned}
{ }_{t} T_{k}^{(0)}\left(s_{0}, \Delta s_{-1}\right)= & \frac{\sum_{j=1}^{k}(-1)^{j}\left(\begin{array}{l}
k \\
j
\end{array}\right) P_{k-1}(j) \frac{s_{j-1}}{\Delta s_{j-1}}}{\sum_{j=0}^{k}(-1)^{j}\left(\begin{array}{l}
k \\
j
\end{array}\right) P_{k-1}(j) \frac{1}{\Delta s_{j-1}}} \\
= & \frac{\sum_{j=1}^{k}(-1)^{j}\left(\begin{array}{l}
k \\
j
\end{array}\right) \mathcal{P}_{k-1}(j-1) \frac{s_{j-1}}{\Delta s_{j-1}}}{\sum_{j=0}^{k}(-1)^{j}\left(\begin{array}{l}
k \\
j
\end{array}\right) \mathcal{P}_{k-1}(j-1) \frac{1}{\Delta s_{j-1}}} \quad k \in \mathbb{N} .
\end{aligned}
$$

The fact that $t$-type transformations are actually $d$-type transformations in disguise can also be deduced from the recursive scheme (3.12) which contains all the other recursive 
schemes of Section 3 as special cases. If we choose the initial conditions in (3.12a) according to $u_{n}=s_{n} / \Delta s_{n-1}$, then (3.12b) implies $G_{1}^{(n)}=\Delta\left[s_{n} / \Delta s_{n-1}\right]=\Delta\left[s_{n-1} / \Delta s_{n-1}\right]$. Thus, (3.12c) with $k \geq 1$ yields the same results as if we had started the recursion with the $d$-type initial conditions $u_{n}=s_{n-1} / \Delta s_{n-1}$.

Numerical cancellation increases the risk of loosing accuracy. Thus, it is probably wiser not to use the $t$-type initial conditions $u_{n}=s_{n} / \Delta s_{n-1}$ in the recursive scheme (3.12) or in any of its special cases, but instead the $d$-type initial conditions $u_{n}=s_{n-1} / \Delta s_{n-1}$.

\section{Richardson-Type Transformations}

Some of the most effective accelerators for logarithmically convergent sequences and series $(\rho=1$ in (4.7)), which abound in scientific applications and which constitute formidable computational problems, can be derived with the help of interpolation theory. Thus, the existence of a function $S$ of a continuous variable is postulated which coincides on a set of discrete arguments $\left\{x_{n}\right\}_{n=0}^{\infty}$ with the elements of the sequence $\left\{s_{n}\right\}_{n=0}^{\infty}$ to be transformed:

$$
S\left(x_{n}\right)=s_{n}, \quad n \in \mathbb{N}_{0} .
$$

This ansatz reduces the convergence acceleration problem to an extrapolation problem. If a finite string $s_{n}, s_{n+1}, \ldots, s_{n+k}$ of $k+1$ sequence elements is known, one can construct an approximation $S_{k}(x)$ to $S(x)$ which satisfies the $k+1$ interpolation conditions $S_{k}\left(x_{n+j}\right)=$ $s_{n+j}$ with $0 \leq j \leq k$. Next, the value of $S_{k}(x)$ has to be determined for $x \rightarrow x_{\infty}$. If this can be done, $S_{k}\left(x_{\infty}\right)$ should provide a better approximation to the limit $s=s_{\infty}$ of the sequence $\left\{s_{n}\right\}_{n=0}^{\infty}$ than the last sequence element $s_{n+k}$ used for its construction.

The most important interpolating functions are either polynomials or rational functions which lead to different convergence algorithm (see for example [132, Section 6]). Here, only polynomial interpolation will be considered. Thus, it is assumed that the $k$-th order approximant $S_{k}(x)$ is a polynomial of degree $k$ in $x$,

$$
S_{k}(x)=\gamma_{0}+\gamma_{1} x+\cdots+\gamma_{k} x^{k}, \quad k \in \mathbb{N},
$$

or equivalently that the model sequence for the Richardson extrapolation scheme 104, whose construction will be sketched below, is a polynomial of degree $k$ in the interpolation points $x_{n}$,

$$
s_{n}=s+\sum_{j=0}^{k-1} c_{j} x_{n}{ }^{j+1}, \quad k \in \mathbb{N}, \quad n \in \mathbb{N}_{0} .
$$

For polynomials, the most natural extrapolation point is $x_{\infty}=0$. Accordingly, we assume that the interpolation points $x_{n}$ satisfy the conditions

$$
\begin{gathered}
x_{0}>x_{1}>\cdots>x_{m}>x_{m+1}>\cdots>0, \\
\lim _{n \rightarrow \infty} x_{n}=0 .
\end{gathered}
$$

The choice $x_{\infty}=0$ implies that the approximation to the limit $s=s_{\infty}$ in (5.3) is to be identified with the constant term $\gamma_{0}$ of the polynomial (5.2).

Several different methods for the construction of interpolating polynomials $S_{k}(x)$ are known (see for example [50, Chapter III]). Since we are only interested in the constant term $\gamma_{0}$ of an interpolating polynomial $S_{k}(x)$ and since in most applications it is desirable to compute simultaneously a whole string of approximants $S_{0}(0), S_{1}(0), S_{2}(0), \ldots$ with increasing polynomial degree, the most economical choice is Neville's scheme [93 for the recursive 
computation of interpolating polynomials. If we set $x=0$ in Neville's scheme, we obtain the following recursive scheme (see for example [31, p. 73] or [132 Eq. (6.1-5)]):

$$
\begin{aligned}
\mathcal{N}_{0}^{(n)}\left(s_{n}, x_{n}\right) & =s_{n}, \quad n \in \mathbb{N}_{0}, \\
\mathcal{N}_{k+1}^{(n)}\left(s_{n}, x_{n}\right) & =\frac{x_{n} \mathcal{N}_{k}^{(n+1)}\left(s_{n+1}, x_{n+1}\right)-x_{n+k+1} \mathcal{N}_{k}^{(n)}\left(s_{n}, x_{n}\right)}{x_{n}-x_{n+k+1}}, \quad k, n \in \mathbb{N}_{0} .
\end{aligned}
$$

In the literature on convergence acceleration, this variant of Neville's scheme is called Richardson extrapolation 104. In 132, Section 7.4] it was shown that this recursive scheme can also be derived with the help of the of the annihilation operator approach described in Section 2 by using divided differences as annihilation operators.

In most applications, Richardson extrapolation is used in combination with the interpolation points $x_{n}=1 /(n+\beta)$ with $\beta>0$. Then, the model sequence (5.3) assumes the following form:

$$
s_{n}=s+\frac{1}{\beta+n} \sum_{j=0}^{k-1} \frac{c_{j}}{(\beta+n)^{j}}, \quad k \in \mathbb{N}, \quad n \in \mathbb{N}_{0} .
$$

This model sequence can be obtained from the model sequence (2.16) for Levin's sequence transformation by setting $\omega_{n}=1 /(\beta+n)$. Consequently, $\mathcal{N}_{k}^{(n)}$ with $x_{n}=1 /(\beta+n)$ is a special Levin transformation and can be expressed as the ratio of two finite sums according to (2.18). Since, however, the denominator of the ratio (2.18) can for $\omega_{n}=1 /(\beta+n)$ be expressed in closed form, $\mathcal{N}_{k}^{(n)}$ possesses an even simpler closed form expression as a finite sum (see for example [91, Lemma 2.1, p. 313] or [132, Eq. (7.3-20)]):

$$
\begin{aligned}
\Lambda_{k}^{(n)}\left(\beta, s_{n}\right) & =\mathcal{N}_{k}^{(n)}\left(s_{n}, 1 /(\beta+n)\right)=\mathcal{L}_{k}^{n}\left(\beta, s_{n}, 1 /(\beta+n)\right) \\
& =(-1)^{k} \sum_{j=0}^{k}(-1)^{j} \frac{(\beta+n+j)^{k}}{j !(k-j) !} s_{n+j}, \quad k, n \in \mathbb{N}_{0} .
\end{aligned}
$$

Moreover, the recursive scheme (5.5) assumes the following form 132 Eq. (7.3-21)]:

$$
\begin{aligned}
\Lambda_{0}^{(n)}\left(\beta, s_{n}\right) & =s_{n}, \quad n \in \mathbb{N}_{0}, \\
\Lambda_{k+1}^{(n)}\left(\beta, s_{n}\right) & =\Lambda_{k}^{(n+1)}\left(\beta, s_{n+1}\right)+\frac{\beta+n}{k+1} \Delta \Lambda_{k}^{(n)}\left(\beta, s_{n}\right), \quad k, n \in \mathbb{N}_{0} .
\end{aligned}
$$

In the case of doubly indexed quantities like $\Lambda_{k}^{(n)}$ it is always assumed that $\Delta$ only acts on the superscript $n$ but not on the subscript $k$, i.e., $\Delta \Lambda_{k}^{(n)}=\Lambda_{k}^{(n+1)}-\Lambda_{k}^{(n)}$.

It is also possible to construct in the case of the sequence transformation (1.1) introduced by Č́žzek, Zamastil, and Skála [48. Eq. (10)] a Richardson-type variant. For that purpose, we set in the model sequence (2.12) $\omega_{n}=1 /\left(n+q_{0}\right)$, where $q_{0}$ is a suitable constant, yielding

$$
s_{n}=s+\sum_{j=0}^{k-1} \frac{c_{j}}{\prod_{m=0}^{j}\left(n+q_{m}\right)}, \quad k, n \in \mathbb{N}_{0}
$$

Thus, $\Delta^{k} \prod_{m=0}^{k-1}\left(n+q_{m}\right)$ is the annihilation operator for the remainder of this model sequence, yielding the following Richardson-type variant of the sequence transformation introduced by Čížek, Zamastil, and Skála [48, Eq. (10)]:

$$
{ }_{R} \mathcal{G}_{k}^{(n)}\left(q_{m}, s_{n}\right)=\mathcal{G}_{k}^{(n)}\left(q_{m}, s_{n}, n+q_{0}\right)=\frac{\Delta^{k}\left[\prod_{m=0}^{k-1}\left(n+q_{m}\right) s_{n}\right]}{\Delta^{k} \prod_{m=0}^{k-1}\left(n+q_{m}\right)}, \quad k, n \in \mathbb{N}_{0}
$$


Of course, this transformation can be expressed as the ratio of two finite sums according to (1.1). However, the denominator of (5.10) can be expressed in closed form. First, we observe that the products in the difference operator representation (5.10) are polynomials of degree $k$ in $n$, satisfying

$$
\prod_{m=0}^{k-1}\left(n+q_{m}\right)=n^{k}+\left(q_{0}+q_{1}+\ldots+q_{k-1}\right) n^{k-1}+\ldots
$$

Next, we use the well known relationship $\Delta^{k} n^{k}=k$ !, which can for instance be derived by iterating the commutator relationship (3.4), and take into account that all polynomials of degree $0,1, \ldots, k-1$ in $n$ are annihilated by $\Delta^{k}$. Thus,

$$
\Delta^{k} \prod_{m=0}^{k-1}\left(n+q_{m}\right)=k !
$$

With the help of (2.14) we then obtain from (5.10):

$$
\begin{aligned}
{ }_{R} \mathcal{G}_{k}^{(n)}\left(q_{m}, s_{n}\right) & =\mathcal{G}_{k}^{(n)}\left(q_{m}, s_{n}, 1 /\left(n+q_{0}\right)\right) \\
& =(-1)^{k} \sum_{j=0}^{k-1}(-1)^{j} \frac{\prod_{m=0}^{k-1}\left(n+j+q_{m}\right)}{j !(k-j) !} s_{n+j}, \quad k, n \in \mathbb{N}_{0} .
\end{aligned}
$$

If we set here $q_{m}=\beta$, we obtain the sequence transformation (5.7) according to

$$
{ }_{R} \mathcal{G}_{k}^{(n)}\left(\beta, s_{n}\right)=\mathcal{G}_{k}^{(n)}\left(\beta, s_{n}, 1 /(\beta+n)\right)=\Lambda_{k}^{(n)}\left(\beta, s_{n}\right), \quad k, n \in \mathbb{N}_{0}
$$

We can derive a recursive scheme for ${ }_{R} \mathcal{G}_{k}^{(n)}\left(q_{m}, s_{n}\right)$ by means of the techniques described in Section 3 For that purpose, we express the numerator of the ratio on the right-hand side of (5.10) as follows:

$$
\begin{aligned}
& Q_{k}^{(n)}=Q_{k}^{(n)}\left(q_{m}, s_{n}\right)=\Delta^{k} Y_{k}^{(n)}, \quad k, n \in \mathbb{N}_{0}, \\
& Y_{k}^{(n)}=Y_{k}^{(n)}\left(q_{m}, s_{n}\right)=\prod_{m=0}^{k-1}\left(n+q_{m}\right) s_{n}, \quad k, n \in \mathbb{N}_{0} .
\end{aligned}
$$

The quantities $Y_{k}^{(n)}$ satisfy for $k \geq 1$ the two-term recursion

$$
Y_{k}^{(n)}=\left(n+q_{k-1}\right) Y_{k-1}^{(n)}
$$

Next, we combine (5.15) - (5.17) with the commutator relationship (3.7), yielding

$$
\begin{aligned}
Q_{k}^{(n)} & =\Delta^{k}\left(n+q_{k-1}\right) Y_{k-1}^{(n)} \\
& =\left[\left(n+k+q_{k-1}\right) E-\left(n+q_{k-1}\right)\right] \Delta^{k-1} Y_{k-1}^{(n)} \\
& =\left(n+k+q_{k-1}\right) Q_{k-1}^{(n+1)}-\left(n+q_{k-1}\right) Q_{k-1}^{(n)}, \quad k \in \mathbb{N}, \quad n \in \mathbb{N}_{0} .
\end{aligned}
$$

Now, we only have to divide the recurrence formula (5.20) for the numerator of (5.10) by the denominator according to 5.12 to obtain the recursive scheme

$$
\begin{aligned}
{ }_{R} \mathcal{G}_{k}^{(0)}\left(q_{m}, s_{n}\right) & =s_{n}, \quad n \in \mathbb{N}_{0}, \\
{ }_{R} \mathcal{G}_{k+1}^{(n)}\left(q_{m}, s_{n}\right) & ={ }_{R} \mathcal{G}_{k}^{(n+1)}\left(q_{m}, s_{n+1}\right)+\frac{n+q_{k}}{k+1} \Delta_{R} \mathcal{G}_{k}^{(n)}\left(q_{m}, s_{n}\right), \quad k, n \in \mathbb{N}_{0} .
\end{aligned}
$$


If we choose in (5.21) $q_{m}=\beta$, we obtain the recursive scheme (5.8) for $\Lambda_{k}^{(n)}\left(\beta, s_{n}\right)$.

By specializing the parameters $q_{m}$ in (5.13) and (5.21), other Richardson-type transformations and their recursive schemes can be obtained. If we choose $q_{m}=\chi+m$, we obtain the Richardson-type variant of the sequence transformation (2.21) [132] Eq. (8.4-11)].

$$
\begin{aligned}
\mathcal{F}_{k}^{(n)}\left(\chi, s_{n}\right) & ={ }_{R} \mathcal{G}_{k}^{(n)}\left(\chi+m, s_{n}\right)=\mathcal{S}_{k}^{(n)}\left(\chi+1, s_{n}, 1 /(\chi+n)\right) \\
& =(-1)^{k} \sum_{j=0}^{k}(-1)^{j} \frac{(\chi+n+j)_{k}}{j !(k-j) !} s_{n+j}, \quad k, n \in \mathbb{N}_{0},
\end{aligned}
$$

and its recursive scheme [132, Eq. (8.4-12)]

$$
\begin{aligned}
\mathcal{F}_{0}^{(n)}\left(\chi, s_{n}\right) & =s_{n}, \quad n \in \mathbb{N}_{0}, \\
\mathcal{F}_{k+1}^{(n)}\left(\chi, s_{n}\right) & =\mathcal{F}_{k}^{(n+1)}\left(\chi, s_{n+1}\right)+\frac{\chi+n+k}{k+1} \Delta \mathcal{F}_{k}^{(n)}\left(\chi, s_{n}\right), \quad k, n \in \mathbb{N}_{0} .
\end{aligned}
$$

If we choose in (5.13) and (5.21) $q_{m}=\zeta-m$, we obtain the Richardson-type variant of the sequence transformation (2.25) [132, Eq. (9.4-11)],

$$
\begin{aligned}
\mathcal{P}_{k}^{(n)}\left(\zeta, s_{n}\right) & ={ }_{R} \mathcal{G}_{k}^{(n)}\left(\zeta-m, s_{n}\right)=\mathcal{M}_{k}^{(n)}\left(\zeta-1, s_{n},-1 /(\zeta+n)\right) \\
& =\sum_{j=0}^{k}(-1)^{j} \frac{(-\zeta-n-j)_{k}}{j !(k-j) !} s_{n+j}, \quad k, n \in \mathbb{N}_{0},
\end{aligned}
$$

and its recursive scheme [132, Eq. (9.4-12)]

$$
\begin{aligned}
\mathcal{P}_{0}^{(n)}\left(\zeta, s_{n}\right) & =s_{n}, \quad n \in \mathbb{N}_{0}, \\
\mathcal{P}_{k+1}^{(n)}\left(\zeta, s_{n}\right) & =\mathcal{P}_{k}^{(n+1)}\left(\zeta, s_{n+1}\right)+\frac{\zeta+n-k}{k+1} \Delta \mathcal{P}_{k}^{(n)}\left(\zeta, s_{n}\right), \quad k, n \in \mathbb{N}_{0} .
\end{aligned}
$$

If we choose in (5.13) and (5.21) $q_{m}=\chi+m / \alpha$, we obtain the Richardson-type variant of the sequence transformation (2.29),

$$
\begin{aligned}
{ }_{R} \mathrm{C}_{k}^{(n)}\left(\alpha, \chi, s_{n}\right) & ={ }_{R} \mathcal{G}_{k}^{(n)}\left(\chi+m / \alpha, s_{n}\right)={ }_{R} \mathrm{e}_{k}^{(n)}\left(\alpha, \chi+1, s_{n}, 1 /(\chi+n)\right) \\
& =\frac{(-1)^{k}}{\alpha^{k}} \sum_{j=0}^{k}(-1)^{j} \frac{(\alpha[\chi+n+j]) k}{j !(k-j) !} s_{n+j}, \quad k, n \in \mathbb{N}_{0},
\end{aligned}
$$

and its recursive scheme

$$
\begin{aligned}
{ }_{R} \mathrm{C}_{0}^{(n)}\left(\alpha, \chi, s_{n}\right) & =s_{n}, \quad n \in \mathbb{N}_{0}, \\
{ }_{R} \mathcal{C}_{k+1}^{(n)}\left(\alpha, \chi, s_{n}\right) & ={ }_{R} \mathcal{C}_{k}^{(n+1)}\left(\alpha, \chi, s_{n+1}\right)+\frac{\chi+n+k / \alpha}{k+1} \Delta_{R} \mathcal{C}_{k}^{(n)}\left(\alpha, \chi, s_{n}\right), \quad k, n \in \mathbb{N}_{0} .
\end{aligned}
$$

Depending upon the value of $\alpha>0,{ }_{R} \mathcal{C}_{k}^{(n)}\left(\alpha, \chi, s_{n}\right)$ interpolates between the Richardsontype transformations $\Lambda_{k}^{(n)}\left(\beta, s_{n}\right)$ and $\mathcal{F}_{k}^{(n)}\left(\chi, s_{n}\right)$. If we choose in (5.26) $\alpha=1$ and compare the resulting expression with (5.22), we find

$$
{ }_{R} \mathcal{C}_{k}^{(n)}\left(1, \chi, s_{n}\right)=\mathcal{F}_{k}^{(n)}\left(\chi, s_{n}\right),
$$

and if we use in (5.26)

$$
\lim _{\alpha \rightarrow \infty} \frac{(\alpha[\chi+n+j])_{k}}{\alpha^{k}}=\lim _{\alpha \rightarrow \infty} \prod_{m=0}^{k-1}(\chi+n+j+m / \alpha)=(\chi+n+j)^{k}
$$


together with $\chi=\beta$ and compare the resulting expression with (5.7), we find

$$
\lim _{\alpha \rightarrow \infty}{ }_{R} \mathfrak{C}_{k}^{(n)}\left(\alpha, \beta, s_{n}\right)=\Lambda_{k}^{(n)}\left(\beta, s_{n}\right) .
$$

\section{Rational Approximants}

In theoretical physics and in applied mathematics, Padé approximants 99] have become the standard tool to overcome problems with slowly convergent or divergent power series. Padé approximants can also be viewed as a special class of sequence transformations since they transform the partial sums

$$
f_{n}(z)=\sum_{\nu=0}^{n} \gamma_{\nu} z^{\nu}, \quad n \in \mathbb{N}_{0}
$$

of a (formal) power series for some function $f$ into a doubly indexed sequence of rational functions (see for example [3, Chapter 1]):

$$
[l / m]_{f}(z)=\frac{P^{[l / m]}(z)}{Q^{[l / m]}(z)}=\frac{p_{0}+p_{1} z+\ldots+p_{l} z^{l}}{1+q_{1} z+\ldots+q_{m} z^{m}}, \quad l, m \in \mathbb{N}_{0} .
$$

We also obtain rational approximants if the $u, t, d$, and $v$ variants considered in Section 4 are applied to the partial sums (6.1). Nevertheless, there are some substantial differences between most sequence transformations and Padé approximants. Levin-type transformations can be computed via their explicit expressions, although it is normally preferable to compute them recursively. The coefficients $p_{0}, \ldots, p_{l}$ and $q_{1}, \ldots, q_{m}$ of the two Padé polynomials $P^{[l / m]}$ and $Q^{[l / m]}$ in (6.2) are, however, chosen in such a way that the Taylor expansion of the ratio $P^{[l / m]}(z) / Q^{[l / m]}(z)$ at $z=0$ agrees with the power series for $f$ as far as possible:

$$
Q^{[l / m]}(z) f(z)-P^{[l / m]}(z)=\mathrm{O}\left(z^{l+m+1}\right), \quad z \rightarrow 0 .
$$

This asymptotic condition leads to a system of $l+m+1$ linear equations. If this system of equations has a solution, it yields the coefficients of the polynomials $P^{[l / m]}(z)$ and $Q^{[l / m]}(z)$ (see for example [3] Chapter 1]).

In most practical applications, Padé approximants are not computed via the defining system of equations, but with the help of recursive algorithms as for example Wynn's epsilon algorithm [153]. Nevertheless, the accuracy-through-order relationship (6.3) guarantees that the Taylor expansion of $[l / m]_{f}(z)$ reproduces the partial sum $f_{l+m}(z)$ from which it was constructed. If a sequence transformation is applied to the partial sums of a (formal) power series, it is by no means obvious whether the resulting expression satisfies an accuracythrough-order relationship of the type of (6.3) (see for example the discussion in [143]).

The accuracy-through-order relationship (6.3) is essential if Padé approximants are to be used for the prediction of unknown series coefficients, which was first described and utilized by Gilewicz [65]. This so-called Padé prediction is based on the fact that a Padé approximant is by construction analytic at the origin. Accordingly, the power series

$$
[l / m]_{f}(z)=\sum_{\nu=0}^{\infty} \gamma_{\nu}^{[l / m]} z^{\nu}
$$

converges in a neighborhood of $z=0$. The accuracy-through-order relationship (6.3) implies $\gamma_{\nu}^{[l / m]}=\gamma_{\nu}$ for $0 \leq \nu \leq l+m$. The remaining coefficients $\gamma_{l+m+\mu+1}^{[l / m]}$ with $\mu \geq 0$ are in general different from the corresponding coefficients $\gamma_{l+m+\mu+1}$ of the power series for $f(z)$. If, however, the Padé approximants $[l / m]_{f}(z)$ converge more rapidly to $f(z)$ than the partial 
sums $f_{l+m}(z)$, from which they are constructed, then the coefficients $\gamma_{l+m+\mu+1}^{[l / m]}$ provide in particularly for smaller values of $\mu$ approximants to the corresponding series coefficients. It is important to note that Padé prediction is not restricted to convergent power series. Thus, Padé prediction can produce useful results even if the power series is a factorially divergent perturbation expansion.

In certain subfields of theoretical physics, the computation of more than a few coefficients of a perturbation expansion can be extremely difficult. Moreover, these coefficients are often affected by comparatively large relative errors. Under such adverse conditions, Padé approximants can be used to make predictions about the leading unknown coefficients of perturbation expansions as well as to make consistency checks for previously calculated coefficients. Further details as well as many examples can be found in $9,36,39,40,41,42,43$,

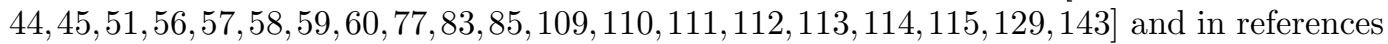
therein. Padé prediction can also be quite helpful in different contexts. For example, Padé prediction techniques developed in [143] were used in 9] to provide numerical evidence that the factorially divergent perturbation expansion for an anharmonic oscillator, whose Hamiltonian is non-Hermitian but $\mathcal{P} \mathcal{T}$-symmetric [7], is a Stieltjes series.

The prediction of unknown power series coefficients is not restricted to Padé approximants. In principle, any other rational approximant, that also satisfies an accuracy-throughorder relationship of the type of (6.3), can be used. It seems that this idea was first formulated by Sidi and Levin [124] and by Brezinski [23. Recently, it was found that Levin-type transformation like (4.35) and (4.36), which satisfy for $k \in \mathbb{N}$ and $n \in \mathbb{N}_{0}$ the following asymptotic order estimates as $z \rightarrow 0$ [149. Eqs. (4.28) and (4.29)],

$$
\begin{aligned}
& f(z)-d_{k}^{(n)}\left(\beta, f_{n}(z)\right)=\mathrm{O}\left(z^{k+n+2}\right), \\
& f(z)-\delta_{k}^{(n)}\left(\beta, f_{n}(z)\right)=\mathrm{O}\left(z^{k+n+2}\right),
\end{aligned}
$$

produce at least in some cases significantly more accurate predictions for unknown power series coefficients than Padé approximants [77,83,142. Accordingly. it should be of interest to analyze not only the rational approximants, which result if Levin-type transformations are applied to power series, but also their accuracy-through-order relationships. In the case of Levin's $t$ transformation, this was already done by Sidi and Levin [124], and the accuracythrough-order relationships for the $u, t, v$, and $d$ variants of $\mathcal{L}_{k}^{(n)}\left(\beta, s_{n}, \omega_{n}\right), \mathcal{S}_{k}^{(n)}\left(\beta, s_{n}, \omega_{n}\right)$, and $\mathcal{M}_{k}^{(n)}\left(\xi, s_{n}, \omega_{n}\right)$ were studied in [136, Section 5.7], albeit by a less elegant method.

In this Section, the $u, t, v$, and $d$ variants of the sequence transformation $\mathcal{G}_{k}^{(n)}\left(q_{m}, s_{n}, \omega_{n}\right)$ introduced by Č́žzek, Zamastil, and Skála [48. Eq. (10)] are applied to (formal) power series and the accuracy-through-order properties of the resulting rational approximants are studied. Since the sequence transformations $\mathcal{L}_{k}^{(n)}\left(\beta, s_{n}, \omega_{n}\right), \mathcal{S}_{k}^{(n)}\left(\beta, s_{n}, \omega_{n}\right), \mathcal{M}_{k}^{(n)}\left(\xi, s_{n}, \omega_{n}\right)$, and $\mathcal{C}_{k}^{(n)}\left(\alpha, \beta, s_{n}, \omega_{n}\right)$ can be obtained from $\mathcal{G}_{k}^{(n)}\left(q_{m}, s_{n}, \omega_{n}\right)$ by specializing the parameters $q_{m}$, all results for ${ }_{u} \mathcal{G}_{k}^{(n)}\left(q_{m}, f_{n}(z)\right),{ }_{t} \mathcal{G}_{k}^{(n)}\left(q_{m}, f_{n}(z)\right),{ }_{d} \mathcal{G}_{k}^{(n)}\left(q_{m}, f_{n}(z)\right)$, and ${ }_{v} \mathcal{G}_{k}^{(n)}\left(q_{m}, f_{n}(z)\right)$ derived here can immediately be translated to the analogous results for the $u, t, d$, and $v$ type variants of the transformations mentioned above.

If we use the partial sums (6.1) of a (formal) power series $f(z)=\sum_{\nu=0}^{n} \gamma_{\nu} z^{\nu}$ as input data, the simple remainder estimates (4.16), (4.18), (4.24), and (4.32) for the $u, t$, $v$, and $d$ variants of $\mathcal{G}_{k}^{(n)}\left(q_{m}, s_{n}, \omega_{n}\right)$ translate to $\omega_{n}=\left(n+q_{0}\right) \gamma_{n} z^{n}, \omega_{n}=\gamma_{n} z^{n}, \omega_{n}=$ $\gamma_{n} \gamma_{n+1} z^{n+1} /\left[\gamma_{n}-z \gamma_{n+1}\right]$, and $\omega_{n}=\gamma_{n+1} z^{n+1}$. Of course, these remainder estimates can only be used if the coefficients of the power series for $f$ satisfy $\gamma_{n} \neq 0$ for all $n \in \mathbb{N}_{0}$. In the following text, this will be tacitly assumed.

If we apply the $u$ variant (4.17) of $\mathcal{G}_{k}^{(n)}\left(q_{m}, s_{n}, \omega_{n}\right)$ to the partial sums (6.1) of the 
(formal) power series for $f$, we obtain:

$$
\begin{aligned}
&{ }_{u} \mathcal{G}_{k}^{(n)}\left(q_{m}, f_{n}(z)\right)=\mathcal{G}_{k}^{(n)}\left(q_{m}, f_{n}(z),\left(n+q_{0}\right) \gamma_{n} z^{n}\right) \\
&= \frac{\sum_{j=0}^{k}(-1)^{j}\left(\begin{array}{l}
k \\
j
\end{array}\right) \prod_{m=1}^{k-1} \frac{\left(n+j+q_{m}\right)}{\left(n+k+q_{m}\right)} \frac{z^{k-j} f_{n+j}(z)}{\left(n+j+q_{0}\right) \gamma_{n+j}}}{\sum_{j=0}^{k}(-1)^{j}\left(\begin{array}{l}
k \\
j
\end{array}\right) \prod_{m=1}^{k-1} \frac{\left(n+j+q_{m}\right)}{\left(n+k+q_{m}\right)} \frac{z^{k-j}}{\left(n+j+q_{0}\right) \gamma_{n+j}}}, \quad k, n \in \mathbb{N}_{0} .
\end{aligned}
$$

In the case of the $t$ variant (4.23) of $\mathcal{G}_{k}^{(n)}\left(q_{m}, s_{n}, \omega_{n}\right)$, we obtain:

$$
\begin{aligned}
{ }_{t} \mathcal{G}_{k}^{(n)}\left(q_{m}, f_{n}(z)\right)=\mathcal{G}_{k}^{(n)}\left(q_{m}, f_{n}(z), \gamma_{n} z^{n}\right) & \\
= & \frac{\sum_{j=0}^{k}(-1)^{j}\left(\begin{array}{l}
k \\
j
\end{array}\right) \prod_{m=1}^{k-1} \frac{\left(n+j+q_{m}\right)}{\left(n+k+q_{m}\right)} \frac{z^{k-j} f_{n+j}(z)}{\gamma_{n+j}}}{\sum_{j=0}^{k}(-1)^{j}\left(\begin{array}{l}
k \\
j
\end{array}\right) \prod_{m=1}^{k-1} \frac{\left(n+j+q_{m}\right)}{\left(n+k+q_{m}\right)} \frac{z^{k-j}}{\gamma_{n+j}}}, \quad k, n \in \mathbb{N}_{0} .
\end{aligned}
$$

In the case of the $d$ variant (4.39) of $\mathcal{G}_{k}^{(n)}\left(q_{m}, s_{n}, \omega_{n}\right)$, we obtain:

$$
\begin{aligned}
{ }_{d} \mathcal{G}_{k}^{(n)}\left(q_{m}, f_{n}(z)\right)=\mathcal{G}_{k}^{(n)}\left(q_{m}, f_{n}(z), \gamma_{n+1} z^{n+1}\right) \\
=\frac{\sum_{j=0}^{k}(-1)^{j}\left(\begin{array}{l}
k \\
j
\end{array}\right) \prod_{m=1}^{k-1} \frac{\left(n+j+q_{m}\right)}{\left(n+k+q_{m}\right)} \frac{z^{k-j} f_{n+j}(z)}{\gamma_{n+j+1}}}{\sum_{j=0}^{k}(-1)^{j}\left(\begin{array}{l}
k \\
j
\end{array}\right) \prod_{m=1}^{k-1} \frac{\left(n+j+q_{m}\right)}{\left(n+k+q_{m}\right)} \frac{z^{k-j}}{\gamma_{n+j+1}}}, \quad k, n \in \mathbb{N}_{0} .
\end{aligned}
$$

The numerators of (6.7) - (6.9) are polynomials of degree $k+n$ in $z$, and the denominators are polynomials of degree $k$ in $z$. For the computation of (6.7) and (6.8), we need the numerical values of the partial sums $f_{n}(z), f_{n+1}(z), \ldots, f_{n+k}(z)$, and for the computation of (6.9), we need the partial sums $f_{n}(z), f_{n+1}(z), \ldots, f_{n+k+1}(z)$.

In the case of the $v$ variant (4.31) of $\mathcal{G}_{k}^{(n)}\left(q_{m}, s_{n}, \omega_{n}\right)$, we obtain:

$$
\begin{aligned}
&{ }_{v} \mathcal{G}_{k}^{(n)}\left(q_{m}, f_{n}(z)\right)=\mathcal{G}_{k}^{(n)}\left(q_{m}, f_{n}(z), \gamma_{n} \gamma_{n+1} z^{n+1} /\left[\gamma_{n}-z \gamma_{n+1}\right]\right) \\
&= \frac{\sum_{j=0}^{k}(-1)^{j}\left(\begin{array}{c}
k \\
j
\end{array}\right) \prod_{m=1}^{k-1} \frac{\left(n+j+q_{m}\right)}{\left(n+k+q_{m}\right)} \frac{z^{k-j}\left(\gamma_{n+j}-z \gamma_{n+j+1}\right) f_{n+j}(z)}{\gamma_{n+j} \gamma_{n+j+1}}}{\sum_{j=0}^{k}(-1)^{j}\left(\begin{array}{c}
k \\
j
\end{array}\right) \prod_{m=1}^{k-1} \frac{\left(n+j+q_{m}\right)}{\left(n+k+q_{m}\right)} \frac{z^{k-j}\left(\gamma_{n+j}-z \gamma_{n+j+1}\right)}{\gamma_{n+j} \gamma_{n+j+1}}}, \quad k, n \in \mathbb{N}_{0} \cdot(6.10)
\end{aligned}
$$

The numerator of this expression is a polynomial of degree $k+n+1$ in $z$, and the denominator is a polynomial of degree $k+1$ in $z$. For its computation, we need the numerical values of the partial sums $f_{n}(z), f_{n+}(z), \ldots, f_{n+k+1}(z)$.

Next, asymptotic order estimates of the type of 6.3) will be constructed for the rational approximants (6.7) - (6.10). Here, it must be taken into account that an accuracy-throughorder relationship does not make any sense if the rational function reproduces exactly the function $f$ represented by the power series. This is for instance the case if $u, t, d$, and $v$ variants of $\mathcal{G}_{k}^{(n)}\left(q_{m}, s_{n}, \omega_{n}\right)$ are applied to the partial sums $\sum_{\nu=0}^{n} z^{\nu}=\left(1-z^{n+1}\right) /(1-z)$ 
of the geometric series. For an analysis of these complications, let us consider the $u$ variant (6.7). If we introduce the remainders of the partial sums (6.1) according to

$$
r_{n}(z)=f_{n}(z)-f(z)=-\sum_{\nu=0}^{\infty} \gamma_{n+\nu+1} z^{n+\nu+1},
$$

then the difference between $f$ and the $u$ variant (6.7) can according to (4.2) be expressed as follows:

$$
\begin{aligned}
& f(z)-{ }_{u} \mathcal{G}_{k}^{(n)}\left(q_{m}, f_{n}(z)\right) \\
& =-z^{k+n} \frac{\sum_{j=0}^{k}(-1)^{j}\left(\begin{array}{l}
k \\
j
\end{array}\right) \prod_{m=1}^{k-1}\left(n+j+q_{m}\right) \frac{r_{n+j}(z)}{\left(n+j+q_{0}\right) \gamma_{n+j} z^{n+j}}}{\sum_{j=0}^{k}(-1)^{j}\left(\begin{array}{l}
k \\
j
\end{array}\right) \prod_{m=1}^{k-1}\left(n+j+q_{m}\right) \frac{z^{k-j}}{\left(n+j+q_{0}\right) \gamma_{n+j}}} .
\end{aligned}
$$

The denominator of this expression is by assumption of order $\mathrm{O}(1)$ as $z \rightarrow 0$. For the derivation of an order estimate of the numerator, we use (2.14) to obtain

$$
\begin{aligned}
& \sum_{j=0}^{k}(-1)^{j}\left(\begin{array}{l}
k \\
j
\end{array}\right) \prod_{m=1}^{k-1}\left(n+j+q_{m}\right) \frac{r_{n+j}(z)}{\left(n+j+q_{0}\right) \gamma_{n+j} z^{n+j}} \\
& \quad=(-1)^{k}\left[\Delta^{k} \prod_{m=1}^{k-1}\left(n+q_{m}\right) \frac{r_{n}(z)}{\left(n+q_{0}\right) \gamma_{n} z^{n}}\right] .
\end{aligned}
$$

We now have to distinguish some special cases.

Let us first assume that the $u$ type remainder estimate $\omega_{n}=\left(n+q_{0}\right) \gamma_{n} z^{n}$ is a perfect remainder estimate according to (4.3). Then, $\Delta^{k}$ acts on a polynomial of degree $k-1$ in $n$, which means that the right-hand side of (6.12) is annihilated. Accordingly, ${ }_{u} \mathcal{G}_{k}^{(n)}\left(q_{m}, f_{n}(z)\right)$ is exact for $k \geq 1$ and an accuracy-through-order relationship of the type of (6.3) makes no sense.

Let us now assume that the $t$ type remainder estimate $\omega_{n}=\gamma_{n} z^{n}$ is a perfect remainder estimate according to (4.3), which is the case if the input data $\left\{f_{n}(z)\right\}_{n=0}^{\infty}$ are the partial sums $\sum_{\nu=0}^{n} z^{\nu}=\left(1-z^{n+1}\right) /(1-z)$ of the geometric series. Then, we again have to distinguish two cases. If $q_{0} \in\left\{q_{1}, \ldots, q_{m}\right\}$, the ratio $\prod_{m=1}^{k-1}\left(n+q_{m}\right) /\left(n+q_{0}\right)$ simplifies to yield a polynomial of degree $k-2$ in $n$ which is annihilated by $\Delta^{k}$. Accordingly, ${ }_{u} \mathcal{G}_{k}^{(n)}\left(q_{m}, f_{n}(z)\right)$ is for $k \geq 1$ exact for the partial sums of the geometric series and an accuracy-through-order relationship makes no sense. If $q_{0} \notin\left\{q_{1}, \ldots, q_{m}\right\}$, the ratio $\prod_{m=1}^{k-1}\left(n+q_{m}\right) /\left(n+q_{0}\right)$ does not simplify to yield a polynomial and is not annihilated by $\Delta^{k}$. Accordingly, ${ }_{u} \mathcal{G}_{k}^{(n)}\left(q_{m}, f_{n}(z)\right)$ is in this case not exact for the geometric series.

The exactness for the geometric series is probably the most fundamental requirement for a sequence transformation in the case of linear convergence $(0<|\rho|<1$ in (4.7)). This follows from Germain-Bonne's formal theory of convergence acceleration 64 and its extension to Levin-type transformations [132, Section 12]. Consequently, it is probably a good idea that to choose $q_{0}$ in in $\omega_{n}=\left(n+q_{0}\right) \Delta s_{n-1}$ according to $q_{0} \in\left\{q_{1}, \ldots, q_{m}\right\}$. An obvious idea would be to choose $q_{0}=q_{1}$. The remainder estimates of the other $u$ type transformations (4.12), (4.13), (4.14), and (4.15) all satisfy this requirement. Accordingly, these $u$ variants are for $k \geq 1$ exact for the geometric series. This is also true for the $t, d$, and $v$ variants (6.8) - 6.10) of $\mathcal{G}_{k}^{(n)}\left(q_{m}, s_{n}, \omega_{n}\right)$.

By analyzing expressions of the type of (6.12), accuracy-through-order relationships for the rational approximants ${ }_{u} \mathcal{G}_{k}^{(n)}\left(q_{m}, f_{n}(z)\right),{ }_{u} \mathcal{G}_{k}^{(n)}\left(q_{m}, f_{n}(z)\right),{ }_{u} \mathcal{G}_{k}^{(n)}\left(q_{m}, f_{n}(z)\right)$, and 
${ }_{u} \mathcal{G}_{k}^{(n)}\left(q_{m}, f_{n}(z)\right)$ can be derived (see for example [136. Section 5.7]). However, it is more elegant to do this via the theory of Padé-type approximants [22].

As is well known, the coefficients of the numerator and the denominator polynomials of a Padé approximants are chosen in such a way that the asymptotic order estimate 6.3. is satisfied, but it is not so well known that generalizations and modifications of Padé approximants can be obtained by suitably modifying the asymptotic condition (6.3). For example, let us consider the rational approximants

$$
(l / m)_{f}(z)=\frac{\mathcal{U}^{(l / m)}(z)}{\mathcal{V}^{(l / m)}(z)}=\frac{u_{0}+u_{1} z+\ldots+u_{l} z^{l}}{v_{0}+v_{1} z+\ldots+v_{m} z^{m}}, \quad l, m \in \mathbb{N}_{0} .
$$

We assume that the two polynomials $\mathfrak{U}^{(l / m)}(z)$ and $\mathcal{V}^{(l / m)}(z)$ are exactly of degrees $l$ and $m$ in $z$, or equivalently that $u_{l} \neq 0$ and $v_{m} \neq 0$. Let us now assume that the $m+1$ coefficients $v_{0}, v_{1}, \ldots, v_{m}$ of the denominator polynomial $\mathcal{V}^{(l / m)}(z)$ are chosen according to some rule. Then, only the $l+1$ coefficients $u_{0}, u_{1}, \ldots, u_{l}$ of the numerator polynomial $u^{(l / m)}(z)$ have to be determined via the modified asymptotic condition

$$
\mathcal{V}^{(l / m)}(z) f(z)-\mathcal{U}^{(l / m)}(z)=\mathrm{O}\left(z^{l+1}\right), \quad z \rightarrow 0,
$$

yielding

$$
\mathcal{U}^{(l / m)}(z)=\sum_{\lambda=0}^{l} v_{\lambda} z^{\lambda} f_{l-\lambda}(z) .
$$

The rational function $(l / m)_{f}(z)$ is a so-called Padé-type approximant. Padé-type approximants and their properties are discussed in a monograph by Brezinski 22 .

Let us now set $m=k$ and $l=k+n$ with $k, n, \in \mathbb{N}_{0}$ in (6.14). Then, (6.16) implies

$$
(k+n / k)_{f}(z)=\frac{\mathcal{u}^{(k+n / k)}(z)}{\mathcal{V}^{(k+n / k)}(z)}=\frac{\sum_{j=0}^{k} v_{j} z^{j} f_{k+n-j}(z)}{\sum_{j=0}^{k} v_{j} z^{j}}=\frac{\sum_{j=0}^{k} v_{k-j} z^{k-j} f_{n+j}(z)}{\sum_{j=0}^{k} v_{k-j} z^{k-j}} .
$$

It follows from [6.7), (6.8), and (6.9) that the rational approximants ${ }_{u} \mathcal{G}_{k}^{(n)}\left(q_{m}, f_{n}(z)\right)$, ${ }_{t} \mathcal{G}_{k}^{(n)}\left(q_{m}, f_{n}(z)\right)$, and ${ }_{d} \mathcal{G}_{k}^{(n)}\left(q_{m}, f_{n}(z)\right)$ possess the following general structure:

$$
\mathbf{T}_{k}^{(n)}(z)=\frac{\sum_{j=0}^{k} \lambda_{j}^{(k, n)} z^{k-j} f_{n+j}(z)}{\sum_{j=0}^{k} \lambda_{j}^{(k, n)} z^{k-j}}=\frac{\sum_{j=0}^{k} \lambda_{k-j}^{(k, n)} z^{j} f_{n+k-j}(z)}{\sum_{j=0}^{k} \lambda_{k-j}^{(k, n)} z^{j}}, \quad k, n \in \mathbb{N}_{0} .
$$

Thus, ${ }_{u} \mathcal{G}_{k}^{(n)}\left(q_{m}, f_{n}(z)\right),{ }_{t} \mathcal{G}_{k}^{(n)}\left(q_{m}, f_{n}(z)\right)$, and ${ }_{d} \mathcal{G}_{k}^{(n)}\left(q_{m}, f_{n}(z)\right)$ are Padé-type approximants of the type of $(k+n / k)_{f}(z)$ with $v_{j}=\lambda_{k-j}^{(k, n)}$.

It is a direct consequence of the defining asymptotic condition (6.15) that the Padé-type approximant $\mathbf{T}_{k}^{(n)}(z)$ satisfies for all $k, n \in \mathbb{N}_{0}$ the accuracy-through-order relationship

$$
f(z)-\mathbf{T}_{k}^{(n)}(z)=\mathrm{O}\left(z^{k+n+1}\right), \quad z \rightarrow 0 .
$$

This implies that the functions ${ }_{u} \mathcal{G}_{k}^{(n)}\left(q_{m}, f_{n}(z)\right)$ and ${ }_{t} \mathcal{G}_{k}^{(n)}\left(q_{m}, f_{n}(z)\right)$ as well as all other $u$ and $t$ type transformations considered in this article satisfy for $k, n \in \mathbb{N}_{0}$ the following 
asymptotic order estimates as $z \rightarrow \infty$ :

$$
\begin{aligned}
f(z)-{ }_{u} \mathcal{G}_{k}^{(n)}\left(q_{m}, f_{n}(z)\right) & =\mathrm{O}\left(z^{k+n+1}\right), \\
f(z)-{ }_{t} \mathcal{G}_{k}^{(n)}\left(q_{m}, f_{n}(z)\right) & =\mathrm{O}\left(z^{k+n+1}\right) .
\end{aligned}
$$

Thus, all coefficients $\gamma_{0}, \gamma_{1}, \ldots, \gamma_{k+n}$ of the power series $f(z)=\sum_{\nu=0}^{\infty} \gamma_{\nu} z^{\nu}$, that are used for the construction of the rational approximants ${ }_{u} \mathcal{G}_{k}^{(n)}\left(q_{m}, f_{n}(z)\right)$ and ${ }_{t} \mathcal{G}_{k}^{(n)}\left(q_{m}, f_{n}(z)\right)$, respectively, are reproduced by a Taylor expansion around $z=0$.

For ${ }_{d} \mathcal{G}_{k}^{(n)}\left(q_{m}, f_{n}(z)\right)$ we obtain the same asymptotic order estimate:

$$
f(z)-{ }_{d} \mathcal{G}_{k}^{(n)}\left(q_{m}, f_{n}(z)\right)=\mathrm{O}\left(z^{k+n+1}\right), \quad k, n \in \mathbb{N}_{0}, \quad z \rightarrow 0,
$$

In the context of the prediction of unknown power series coefficients, this is a highly unwelcome result: For the computation of ${ }_{d} \mathcal{G}_{k}^{(n)}\left(q_{m}, f_{n}(z)\right)$ we need the series coefficients $\gamma_{0}, \gamma_{1}, \ldots, \gamma_{n+k+1}$. Thus, the order term $\mathrm{O}\left(z^{k+n+1}\right)$ implies that a Taylor expansion of ${ }_{d} \mathcal{G}_{k}^{(n)}\left(q_{m}, f_{n}(z)\right)$ does not reproduce all coefficients used for its construction. Moreover, the order estimates (6.5) and (6.6), which were derived in [149] by directly analyzing the corresponding expressions without using the theory of Padé-type approximants, indicate that we should instead get the order estimate

$$
f(z)-{ }_{d} \mathcal{G}_{k}^{(n)}\left(q_{m}, f_{n}(z)\right)=\mathrm{O}\left(z^{k+n+2}\right), \quad k \in \mathbb{N}, \quad n \in \mathbb{N}_{0}, \quad z \rightarrow 0 .
$$

It is indeed possible to derive this seemingly irregular accuracy-through-order relationship by analyzing the Padé-type approximant $\mathbf{T}_{k}^{(n)}(z)$ more carefully. For that purpose, we rewrite (6.18) as follows:

$$
\mathbf{T}_{k}^{(n)}(z)=f(z)-z^{k+n+1} \frac{\sum_{j=0}^{k} \lambda_{j}^{(k, n)} \sum_{\nu=0}^{\infty} \gamma_{n+j+\nu+1} z^{\nu}}{\sum_{j=0}^{k} \lambda_{j}^{(k, n)} z^{k-j}} .
$$

The denominator on the right-hand side is by assumption of order $\mathrm{O}(1)$ as $z \rightarrow 0$. Accordingly, the asymptotic estimate (6.19) is normally optimal, and the improved asymptotic estimate (6.23) can only hold if the $z$-independent part of the numerator vanishes, or equivalently if $\sum_{j=0}^{k} \lambda_{j}^{(k, n)} \gamma_{n+j+1}=0$. For essentially arbitrary coefficients $\lambda_{j}^{(k, n)}$ this is certainly not true. However, in the case of all $d$ type transformations of this article we have $\lambda_{j}^{(k, n)}=(-1)^{j}\left(\begin{array}{l}k \\ j\end{array}\right) P_{k-1}(n+j) / \gamma_{n+j+1}$, where $P_{k-1}(n)$ is a suitable polynomial of degree $k-1$ in $n$. Then, we have for $k \geq 1$

$$
\sum_{j=0}^{k} \lambda_{j}^{(k, n)} \gamma_{n+j+1}=\sum_{j=0}^{k}(-1)^{j}\left(\begin{array}{l}
k \\
j
\end{array}\right) P_{k-1}(n+j)=(-1)^{k} \Delta^{k} P_{k-1}(n)=0 .
$$

This proofs the refined accuracy-through-order relationship (6.23).

It follows from (6.10) that the rational approximant ${ }_{v} \mathcal{G}_{k}^{(n)}\left(q_{m}, f_{n}(z)\right)$ possesses like all other $v$ type transformations of this article the following general structure:

$$
\mathbf{V}_{k}^{(n)}(z)=\frac{\sum_{j=0}^{k} \lambda_{j}^{(k, n)} z^{k-j} f_{n+j}(z)+z \sum_{j=0}^{k} \mu_{j}^{(k, n)} z^{k-j} f_{n+j}(z)}{\sum_{j=0}^{k} \lambda_{j}^{(k, n)} z^{k-j}+z \sum_{j=0}^{k} \mu_{j}^{(k, n)} z^{k-j}}, \quad k, n \in \mathbb{N}_{0} .
$$


Comparison with (6.18) shows that $\mathbf{V}_{k}^{(n)}(z)$ is no Padé-type approximant. Accordingly, the defining asymptotic condition (6.15) of Padé-type approximants cannot be applied. Fortunately, an analogous asymptotic order estimate for $\mathbf{V}_{k}^{(n)}(z)$ can be derived easily. For that purpose, we rewrite (6.26) as follows:

$$
\begin{aligned}
\mathbf{V}_{k}^{(n)}(z)= & f(z) \\
-z^{k+n+1} & \frac{\sum_{j=0}^{k} \lambda_{j}^{(k, n)} \sum_{\nu=0}^{\infty} \gamma_{n+j+\nu+1} z^{\nu}+z \sum_{j=0}^{k} \mu_{j}^{(k, n)} \sum_{\nu=0}^{\infty} \gamma_{n+j+\nu+1} z^{\nu}}{\sum_{j=0}^{k} \lambda_{j}^{(k, n)} z^{k-j}+z \sum_{j=0}^{k} \mu_{j}^{(k, n)} z^{k-j}} .
\end{aligned}
$$

The denominator on the right-hand side is by assumption of order $\mathrm{O}(1)$ as $z \rightarrow 0$. Accordingly, we obtain the asymptotic order estimate

$$
f(z)-\mathbf{V}_{k}^{(n)}(z)=\mathrm{O}\left(z^{k+n+1}\right), \quad k, n \in \mathbb{N}_{0}, \quad z \rightarrow 0,
$$

which implies

$$
f(z)-{ }_{v} \mathcal{G}_{k}^{(n)}\left(q_{m}, f_{n}(z)\right)=\mathrm{O}\left(z^{k+n+1}\right), \quad k, n \in \mathbb{N}_{0}, \quad z \rightarrow 0 .
$$

Now, we have the same problem as in the case of the suboptimal order estimate (6.22) for ${ }_{d} \mathcal{G}_{k}^{(n)}\left(q_{m}, f_{n}(z)\right)$ : The order term $\mathrm{O}\left(z^{k+n+1}\right)$ in (6.29) implies that a Taylor expansion of ${ }_{v} \mathcal{G}_{k}^{(n)}\left(q_{m}, f_{n}(z)\right)$ reproduces only $\gamma_{0}, \gamma_{1}, \ldots, \gamma_{n+k}$, whereas $\gamma_{0}, \gamma_{1}, \ldots, \gamma_{n+k+1}$ are needed for the computation of ${ }_{v} \mathcal{G}_{k}^{(n)}\left(q_{m}, f_{n}(z)\right)$. Thus, we need instead the order estimate

$$
f(z)-{ }_{v} \mathcal{G}_{k}^{(n)}\left(q_{m}, f_{n}(z)\right)=\mathrm{O}\left(z^{k+n+2}\right), \quad k \in \mathbb{N}, \quad n \in \mathbb{N}_{0}, \quad z \rightarrow 0 .
$$

This refined asymptotic estimate can only be true if the $z$-independent part of the first numerator sum in (6.27) vanishes, or equivalently if $\sum_{j=0}^{k} \lambda_{j}^{(k, n)} \gamma_{n+j+1}=0$. For essentially arbitrary coefficients $\lambda_{j}^{(k, n)}$ this is certainly not true. However, in the case of the $v$ type transformations of this article we have just like in the case of the $d$ type transformations $\lambda_{j}^{(k, n)}=(-1)^{j}\left(\begin{array}{c}k \\ j\end{array}\right) P_{k-1}(n+j) / \gamma_{n+j+1}$ and $\mu_{j}^{(k, n)}=(-1)^{j+1}\left(\begin{array}{l}k \\ j\end{array}\right) P_{k-1}(n+j) / \gamma_{n+j}$, where $P_{k-1}(n)$ is a suitable polynomial of degree $k-1$ in $n$. Thus, 6.25) holds which proves the accuracy-through-order relationship (6.30).

In Section 4 it was mentioned that in some cases asymptotic expressions $a_{n}^{(\infty)}$ for the terms $a_{n}$ of an infinite series $s=\sum_{\nu=0}^{\infty} a_{\nu}$ are known which reproduce the leading order asymptotics of $a_{n}$ as $n \rightarrow \infty$, and that these asymptotic expressions can also be used in the simple remainder estimates (4.11), (4.18), (4.24), and (4.32) since they also reproduce the leading order asymptotics of the remainders $r_{n}=s_{n}-s$ as $n \rightarrow \infty$.

Thus, we now assume that asymptotic expressions $\gamma_{n}^{(\infty)}$ are known that reproduce the leading order asymptotics of the coefficients of the power series $f(z)=\sum_{\nu=0}^{\infty} \gamma_{\nu} z^{\nu}$ according to

$$
\gamma_{n}=\gamma_{n}^{(\infty)}[c+\mathrm{O}(1 / n)], \quad c \neq 0, \quad n \rightarrow \infty .
$$

If we use in $\mathcal{G}_{k}^{(n)}\left(q_{m}, s_{n}, \omega_{n}\right)$ the $u, t, v$, and $d$ type remainder estimates $\omega_{n}=\left(n+q_{0}\right) \gamma_{n}^{(\infty)} z^{n}$, $\omega_{n}=\gamma_{n}^{(\infty)} z^{n}, \omega_{n}=\gamma_{n}^{(\infty)} \gamma_{n+1}^{(\infty)} z^{n+1} /\left[\gamma_{n}^{(\infty)}-z \gamma_{n+1}^{(\infty)}\right]$, and $\omega_{n}=\gamma_{n+1}^{(\infty)} z^{n+1}$, we obtain rational approximants which closely resemble the $u, t, v$, and $d$ variants (6.7), (6.8), (6.9), and (6.10), and which are also special cases of the rational functions $\mathbf{T}_{k}^{(n)}(z)$ and $\mathbf{V}_{k}^{(n)}(z)$ defined in 
(6.18) and (6.26), respectively. Consequently, these rational approximants satisfy for all $k, n \in \mathbb{N}_{0}$ the following asymptotic estimates as $z \rightarrow 0$ :

$$
\begin{aligned}
f(z)-\mathcal{G}_{k}^{(n)}\left(q_{m}, f_{n}(z),\left(n+q_{0}\right) \gamma_{n}^{(\infty)} z^{n}\right) & =\mathrm{O}\left(z^{k+n+1}\right), \\
f(z)-\mathcal{G}_{k}^{(n)}\left(q_{m}, f_{n}(z), \gamma_{n}^{(\infty)} z^{n}\right) & =\mathrm{O}\left(z^{k+n+1}\right), \\
f(z)-\mathcal{G}_{k}^{(n)}\left(q_{m}, f_{n}(z), \gamma_{n+1}^{(\infty)} z^{n}\right) & =\mathrm{O}\left(z^{k+n+1}\right), \\
f(z)-\mathcal{G}_{k}^{(n)}\left(q_{m}, f_{n}(z), \gamma_{n}^{(\infty)} \gamma_{n+1}^{(\infty)} z^{n+1} /\left[\gamma_{n}^{(\infty)}-z \gamma_{n+1}^{(\infty)}\right]\right) & =\mathrm{O}\left(z^{k+n+1}\right) .
\end{aligned}
$$

Accordingly, all coefficients $\gamma_{0}, \gamma_{1}, \ldots, \gamma_{k+n}$ of the power series for $f(z)$, which were used for the construction of these rational approximants. are reproduced by Taylor expansion.

Improved asymptotic estimates of the type of (6.23) or 6.30) for the $d$ and $v$ type variants do not hold here. The reason is that the coefficients $\lambda_{j}^{(k, n)}$ in (6.18) and (6.26) now satisfy $\lambda_{j}^{(k, n)}=(-1)^{j}\left(\begin{array}{c}k \\ j\end{array}\right) P_{k-1}(n+j) / \gamma_{n+j+1}^{(\infty)}$. In general, we have $\gamma_{n+j+1}^{(\infty)} \neq \gamma_{n+j+1}$, which implies that $\sum_{j=0}^{k} \lambda_{j}^{(k, n)} \gamma_{n+j+1}=0$ does not hold.

If we use in $\mathcal{G}_{k}^{(n)}\left(q_{m}, s_{n}, \omega_{n}\right)$ the remainder estimates $\omega_{n}=\left(n+q_{0}\right) \gamma_{n}^{(\infty)} z^{n}, \omega_{n}=\gamma_{n}^{(\infty)} z^{n}$, $\omega_{n}=\gamma_{n}^{(\infty)} \gamma_{n+1}^{(\infty)} z^{n+1} /\left[\gamma_{n}^{(\infty)}-z \gamma_{n+1}^{(\infty)}\right]$, and $\omega_{n}=\gamma_{n+1}^{(\infty)} z^{n+1}$, the poles of the resulting rational approximants are determined by the parameters $q_{m}$ in the products $\prod_{m=1}^{k-1}\left(n+q_{m}\right)$ and by the remainder estimates, but they do not depend on the coefficients $\gamma_{n}$ of the power series for $f$. This highlights once more the crucial importance of the remainder estimates for the success or the failure of Levin-type transformations. In contrast, both the numerator and the denominator coefficients of a Padé approximant $[l / m]_{f}(z)$ depend via (6.3) on the coefficients of the partial sum $f_{l+m}(z)$ which was used for its construction.

\section{Summary and Outlook}

Levin [90] deserves credit for realizing that the efficiency of convergence acceleration and summation processes can be enhanced considerably by using as input data not only the elements of the sequence $\left\{s_{n}\right\}_{n=0}^{\infty}$ to be transformed, but also explicit estimates $\left\{\omega_{n}\right\}_{n=0}^{\infty}$ for the truncation errors of this sequence.

If the input data are the partial sums of an infinite series, and if sufficiently simple analytical expressions for the terms of this series are known, then it is possible to derive analytical estimates for the truncation errors of these series. In principle, the use of specially designed analytical remainder estimates would be highly desirable, although the resulting expressions are no longer generally applicable sequence transformations, but rather (optimized) approximation schemes for specific problems (see for instance the discussion in [145]).

However, convergence acceleration and summation methods are needed most if only relatively few elements of a slowly convergent or divergent sequence are available, and if apart from the numerical values of the input data virtually nothing is known. This is a scenario which happens only too often if we try to sum divergent perturbation expansions of quantum physics. In such a situation there is obviously no chance of constructing analytical expressions for remainder estimates. Instead, we must construct the remainder estimates from the numerical values of the input data via simple rules. Fortunately, the simple remainder estimates proposed by Levin [90] and later Smith and Ford [126], which are discussed in Section 4 normally do the job. In spite of their simplicity, they often work remarkably well.

If we approximate the remainder $r_{n}$ of a sequence element $s_{n}$ by the product $\omega_{n} z_{n}$ according to (2.8), where $z_{n}$ is a so-called correction term, then we should take into account that our approximation scheme actually has two degrees of freedom. Levin 90] originally assumed that $z_{n}$ is a truncated inverse power series according to (2.16). This is certainly a very natural idea, and it leads to a very powerful sequence transformation.

Ernst Joachim Weniger: A New Levin-Type Sequence Transformation. I. 
Nevertheless, in some cases Levin's transformation fails horribly for reasons which we do not completely understand. For example, it was found in 148,149 that Levin's transformation diverges if it is used for the summation of the perturbation expansions for the ground state energies of the anharmonic oscillators (compare also [134 Table 2] or the discussion in [136] Section 10.7]). A similar divergence of Levin's transformation was observed by Ćížek, Zamastil, and Skála [48, p. 965] in the case of the hydrogen atom in an external magnetic field. Fortunately, Levin's choice for $z_{n}$ is not the only possibility, and at least for some problems, alternative correction terms produce significantly better results. For example, the so-called delta transformation defined in (4.36) is based on the assumption that $z_{n}$ is a truncated factorial series according to (2.19). As mentioned before, this delta transformation is a very effective transformation for slowly convergent and divergent alternating series. In particular, it produces very good summation results both in the case of the anharmonic oscillators [133 134, 136, 139, 141,148, 149] as well as in the case of the hydrogen atom in an external magnetic field [48, Tables I and II].

The sequence transformation $\mathcal{G}_{k}^{(n)}\left(q_{m}, s_{n}, \omega_{n}\right)$ introduced by Čížek, Zamastil, and Skála 48. permits a unified treatment of the mathematical properties of all sequence transformations, whose correction terms are annihilated by difference operators of the type of

$$
\hat{T}=\Delta^{k} P_{k-1}(n)
$$

Here, $P_{k-1}(n)$ is a suitable polynomial of degree $k-1$ in $n$ that can be obtained by specializing the parameters $q_{m}$ in $\prod_{m=1}^{k-1}\left(n+q_{m}\right)$. All Levin-type transformations considered in this article belong to this class of sequence transformations. Consequently, all their mathematical properties such as explicit expressions (Sections 21 and 5), recurrence formulas (Section 3), and accuracy-through-order properties (Section [6) can be deduced from the corresponding properties of $\mathcal{G}_{k}^{(n)}\left(q_{m}, s_{n}, \omega_{n}\right)$ by specializing the parameters $q_{m}$.

In addition, new sequence transformations can be constructed by specializing the parameters $q_{m}$ in $\mathcal{G}_{k}^{(n)}\left(q_{m}, s_{n}, \omega_{n}\right)$. For example, Č́ížek, Zamastil, and Skála 48, Tables I and II] found that in the case of the hydrogen atom in an external magnetic field at least for some coupling constants better summation results can be obtained by choosing $q_{m}=m^{2}$ instead of choosing $q_{m}=m$ which yields the delta transformation with $\beta=1$. Such a quadratic dependence of the parameters $q_{m}$ on $m$ leads to a completely new sequence transformation. Thus, the sequence transformation $\mathcal{G}_{k}^{(n)}\left(q_{m}, s_{n}, \omega_{n}\right)$ introduced by Čížek, Zamastil, and Skála [4] does not only permit a unification of already known transformations, but it also opens up the path for promising new research.

As discussed in more details in the following article [146], our current level of theoretical understanding does not permit to predict which one of the numerous variants of $\mathcal{G}_{k}^{(n)}\left(q_{m}, s_{n}, \omega_{n}\right)$ will give best results for a given convergence acceleration or summation problem. So, if we for example use one of the numerous Levin-type transformation for the summation of a divergent perturbation expansion, we are essentially conducting a numerical experiment. As every good experimentalist knows, a single experiment is only rarely able to provide a definite answer. Normally, a whole set of related experiments is needed to obtain convincing evidence. Of course, this applies also to our numerical experiments. Therefore, we should not insist with a quasi-religious zeal on using only a single (Levin-type) transformation which we for some reason may prefer. Instead, it is usually a much better idea to compare the performance of several different transformations.

Levin-type transformations are not only very powerful but also very flexible. Experience shows that they can handle successfully a large variety of different convergence acceleration or summation problems. This is a direct consequence of the fact that the ansatz $\omega_{n} z_{n}$ for $r_{n}$ according to (2.8) has two degrees of freedom which allows a considerable amount of finetuning. Nevertheless, Levin-type transformations are not a cure for all evils. Consequently, 
a good experimentalist should also take into account the possibility that Levin-type transformations may not work at all or that other transformations could produce better results.

\section{References}

[1] Aitken, A. C., "On Bernoulli's numerical solution of algebraic equations," Proc. Roy. Soc. Edinburgh 46, 289 - 305 (1926).

[2] Aksenov, S. V., Savageau, M. A., Jentschura, U. D., Becher, J., Soff, G., and Mohr, P. J., "Application of the combined nonlinear-condensation transformation to problems in statistical analysis and theoretical physics," Comput. Phys. Commun. 150, 1 -20 (2003).

[3] Baker, G. A., Jr. and Graves-Morris, P., Padé Approximants, 2nd edition (Cambridge U. P., Cambridge, 1996).

[4] Bar-Shalom, A., Klapisch, M., and Oreg, J., "Phase-amplitude algorithms for atomic continuum orbitals and radial integrals," Comput. Phys. Commun. 93, 21 - 32 (1996).

[5] Bar-Shalom, A., Klapisch, M., and Oreg, J., "Hullac, an integrated computer package for atomic processes in plasmas," J. Quant. Spect. Rad. Trans. 71, $169-188$ (2001).

[6] Belkić, D., "New hybrid non-linear transformations of divergent perturbation series for quadratic Zeeman effects," J. Phys. A 22, 3003 - 3010 (1989).

[7] Bender, C. M. and Dunne, G. V., "Large-order perturbation theory for a nonHermitian PJ-symmetric Hamiltonian," J. Math. Phys. 40, 4616 - 4621 (1999).

[8] Bender, C. M. and Orszag, S. A., Advanced Mathematical Methods for Scientists and Engineers, New York (McGraw-Hill, 1978).

[9] Bender, C. M. and Weniger, E. J., "Numerical evidence that the perturbation expansion for a non-Hermitian $\mathcal{P} \mathcal{T}$-symmetric Hamiltonian is Stieltjes," J. Math. Phys. 42, $2167-2183$ (2001).

[10] Bender, C. M. and Wu, T. T., "Anharmonic oscillator," Phys. Rev. 184, 1231 - 1260 (1969).

[11] Bender, C. M. and Wu, T. T., "Large-order behavior of perturbation theory," Phys. Rev. Lett. 27, $461-465$ (1971).

[12] Bender, C. M. and Wu, T. T., "Anharmonic oscillator. II. a study in perturbation theory in large order," Phys. Rev. D 7, 1620 - 1636 (1973).

[13] Bhattacharya, R., Roy, D., and Bhowmick, S., "On the regularity of the Levin $u$ transform," Comput. Phys. Commun. 55, 297 - 301 (1989).

[14] Bhattacharya, R., Roy, D., and Bhowmick, S., "Rational interpolation using LevinWeniger transforms," Comput. Phys. Commun. 101, 213 - 222 (1997).

[15] Borel, E., "Mémoires sur les séries divergentes," Ann. Sci. Ec. Norm. Sup. Paris 16, $9-136$ (1899).

[16] Borel, E., Leçons sur les Séries Divergentes, 2nd edition (Gautier-Villars, Paris, 1928). Reprinted by Éditions Jacques Gabay (Paris, 1988). English translation by C. L. Critchfield and A. Vakar, Lectures on Divergent Series, Translation LA-6140-TR (Los Alamos Scientific Laboratory, Los Alamos, 1975). 
[17] Borghi, R. and Santarsiero, M., "Summing Lax series for nonparaxial beam propagation," Opt. Lett. 28, $774-776$ (2003).

[18] Bouferguene, A. and Fares, M., "Convergence accelerators in the computation of molecular integrals over Slater-type basis functions in the two-range one-center expansion method," Phys. Rev. E 49, 3462 - 3474 (1994).

[19] Brezinski, C., Accélération de la Convergence en Analyse Numérique (Springer-Verlag, Berlin, 1977).

[20] Brezinski, C., Algorithmes d'Accélération de la Convergence - Étude Numérique (Éditions Technip, Paris, 1978).

[21] Brezinski, C., "A general extrapolation algorithm," Numer. Math. 35, 175 - 180 (1980).

[22] Brezinski, C., Padé-Type Approximation and General Orthogonal Polynomials (Birkhäuser, Basel, 1980).

[23] Brezinski, C., "Prediction properties of some extrapolation methods," Appl. Numer. Math. 1, $457-462$ (1985).

[24] Brezinski, C., History of Continued Fractions and Padé Approximants (SpringerVerlag, Berlin, 1991).

[25] Brezinski, C., "Extrapolation algorithms and Padé approximations: A historical survey," Appl. Numer. Math. 20, 299 - 318 (1996).

[26] Brezinski, C., Projection Methods for Systems of Equations (Elsevier, Amsterdam, 1997).

[27] Brezinski, C., "Error estimates and convergence acceleration," in Bulgak, H. and Zenger, C. (Editors), Error Control and Adaptivity in Scientific Computing, 87 - 94 (Kluwer, Dordrecht, 1999).

[28] Brezinski, C., "Difference and differential equations, and convergence acceleration algorithms," CRM Proc. Lect. Notes 25, 53 - 63 (2000).

[29] Brezinski, C., "Convergence acceleration during the 20th century," J. Comput. Appl. Math. 122, 1 - 21 (2000). Reprinted in Brezinski, C. (Editor), Numerical Analysis 2000, Vol. 2: Interpolation and Extrapolation, 1 - 21 (Elsevier, Amsterdam, 2000).

[30] Brezinski, C. and Matos, A. C., "A derivation of extrapolation algorithms based on error estimates," J. Comput. Appl. Math. 66, 5 - 26 (1996).

[31] Brezinski, C. and Redivo Zaglia, M., Extrapolation Methods (North-Holland, Amsterdam, 1991).

[32] Brezinski, C. and Redivo Zaglia, M., "A general extrapolation procedure revisited," Adv. Comput. Math. 2, $461-477$ (1994).

[33] Brezinski, C. and Redivo Zaglia, M., "On the kernel of sequence transformations," Appl. Numer. Math. 16, 239 - 244 (1994).

[34] Brezinski, C. and Redivo Zaglia, M., "Vector and matrix sequence transformations based on biorthogonality," Appl. Numer. Math. 21, 353 - 373 (1996).

[35] Brezinski, C. and Salam, A., "Matrix and vector sequence transformations revisited," Proc. Edinb. Math. Soc. 38, 495 - 510 (1995). 
[36] Brodsky, S. J., Ellis, J., Gardi, E., Karliner, M., and Samuel, M. A., "Padé approximants, optimal renormalization scales, and momentum flow in Feynman diagrams," Phys. Rev. D 56, 6980 - 6992 (1998).

[37] Büsse, G., Kleindienst, H., and Lüchow, A., "Nonrelativistic energies for the Be atom: Double-linked Hylleraas-CI calculation," Int. J. Quantum Chem. 66, 241 - 247 (1998).

[38] Carlson, B. C., Special Functions of Applied Mathematics (Academic Press, New York, 1977).

[39] Chishtie, F. and Elias, V., "Renormalization-scale invariance, minimal sensitivity, and the inclusive hadronic decays of a 115 GeV Higgs particle," Phys. Rev. D 64, 016007-1 -016007-6 (2001).

[40] Chishtie, F., Elias, V., Miransky, V. A., and Steele, T. G., "Padé-summation approach to QCD $\beta$-function infrared properties," Prog. Theor. Phys. 104, $603-631$ (2000).

[41] Chishtie, F., Elias, V., and Steele, T. G., "Asymptotic Padé-approximant predictions for renormalization-group functions of massive $\phi^{4}$ scalar field theory," Phys. Lett. B 446, $267-271$ (1999).

[42] Chishtie, F., Elias, V., and Steele, T. G., "Asymptotic Padé-approximant method and QCD current correlation functions," Phys. Rev. D 59, 10513-1 - 10513-10 (1999).

[43] Chishtie, F., Elias, V., and Steele, T. G., "Padé-improvement of CP-odd Higgs decay rate into two gluons," J. Phys. G 26, 93 - 98 (2000).

[44] Chishtie, F., Elias, V., and Steele, T. G., "Padé-improved estimates of hadronic Higgs decay rates," J. Phys. G 26, 1239 - 1253 (2000).

[45] Chishtie, F., Elias, V., and Steele, T. G., "Padé estimate of QCD's infrared boundary," Phys. Lett. B 514, 279 - 283 (2001).

[46] Čížek, J., Vinette, F., and Weniger, E. J., "Examples on the use of symbolic computation in physics and chemistry: Applications of the inner projection technique and of a new summation method for divergent series," Int. J. Quantum Chem. Symp. 25, $209-223$ (1991).

[47] Č́ižek, J., Vinette, F., and Weniger, E. J., "On the use of the symbolic language Maple in physics and chemistry: Several examples," in de Groot, R. A. and Nadrchal, J. (Editors), Proceedings of the Fourth International Conference on Computational Physics PHYSICS COMPUTING '92, 31 - 44 (World Scientific, Singapore, 1993).

[48] Čížek, J., Zamastil, J., and Skála, L., "New summation technique for rapidly divergent perturbation series. Hydrogen atom in magnetic field," J. Math. Phys. 44, $962-968$ (2003).

[49] Corless, R. M., Essential Maple 7: An Introduction for Scientific Programmers (Springer-Verlag, New York, 2002).

[50] Cuyt, A. and Wuytack, L., Nonlinear Methods in Numerical Analysis (North-Holland, Amsterdam, 1987).

[51] Cvetič, G. and Yu, J.-Y., "Borel-Padé vs Borel-Weniger method: A QED and a QCD example," Mod. Phys. Lett. A 15, 1227 - 1235 (2000).

[52] de Prunelé, E., "Power series with rational coefficients for two-electron atom energies," Int. J. Quantum Chem. 63, 1079 - 1089 (1997). 
[53] Dolovich, A. T. and Brodland, G. W., "Convergence acceleration for iterative finiteelement methods," J. Engin. Mech. 121, 1 - 6 (1995).

[54] Dyson, D. J., "Divergence of perturbation theory in quantum electrodynamics," Phys. Rev. 85, $32-33$ (1952).

[55] Edgal, U. F. and Huber, D. L., "Improved analytical investigation of the hard particle system: Two- and three-dimensional cases," J. Chem. Phys. 108, 1578 - 1586 (1998).

[56] Elias, V., Steele, T. G., Chishtie, F., Migneron, R., and Sprague, K., "Padé improvement of QCD running coupling constants, running masses, Higgs decay rates, and scalar channel sum rules," Phys. Rev. D 58, 116007-1 - 116007-15 (1998).

[57] Ellis, J., Gardi, E., Karliner, M., and Samuel, M. A., "Padé approximants, Borel transforms and renormalons: The Bjorken sum rule as a case study," Phys. Lett. B 366, $268-275$ (1996).

[58] Ellis, J., Gardi, E., Karliner, M., and Samuel, M. A., "Renormalization-scheme dependence of Padé summation in QCD," Phys. Rev. D 54, 6986 - 6996 (1996).

[59] Ellis, J., Jack, I., Jones, D. R. T., Karliner, M., and Samuel, M. A., "Asymptotic Padé approximant predictions: Up to five loops in QCD and SQCD," Phys. Rev. D 57, $2665-2675$ (1998).

[60] Ellis, J., Karliner, M., and Samuel, M. A., "A prediction for the 4-loop $\beta$ function in QCD," Phys. Lett. B 400, 176 - 181 (1997).

[61] Fessler, T., Ford, W. F., and Smith, D. A., "HURRY: An acceleration algorithm for scalar sequences and series," ACM Trans. Math. Software 9, $346-354$ (1983).

[62] Fischer, J., "On the role of power expansions in quantum field theory," Int. J. Mod. Phys. A 12, 3625 - 3663 (1997).

[63] Ford, W. F. and Sidi, A., "An algorithm for a generalization of the Richardson extrapolation process," SIAM J. Numer. Anal. 24, 1212 - 1232 (1987).

[64] Germain-Bonne, B., "Transformations de suites," Rev. Française Automat. Informat. Rech. Operat. 7, $84-90$ (1973).

[65] Gilewicz, J., "Numerical detection of the best Padé approximant and determination of the Fourier coefficients of the insufficiently sampled functions," in Graves-Morris, P. R. (Editor), Padé Approximants and Their Applications, 99 - 103 (Academic Press, London, 1973).

[66] Graffi, S. and Grecchi, V., "Borel summability and indeterminacy of the Stieltjes moment problem: Application to the anharmonic oscillators," J. Math. Phys. 19, $1002-1006$ (1978).

[67] Grotendorst, J., "A Maple programs for converting series expansions to rational functions using the Levin transformation," Comput. Phys. Commun. 55, 325 - 335 (1989).

[68] Grotendorst, J. and Steinborn, E. O., "Use of nonlinear convergence accelerators for the efficient evaluation of GTO molecular integrals," J. Chem. Phys. 84, 5617 - 5623 (1986).

[69] Grotendorst, J., Weniger, E. J., and Steinborn, E. O., "Efficient evaluation of infiniteseries representations for overlap, two-center nuclear attraction, and coulomb integrals using nonlinear convergence accelerators," Phys. Rev. A 33, 3706 - 3726 (1986). 
[70] Håvie, T., "Generalized Neville type extrapolation schemes," BIT 19, $204-213$ (1979).

[71] Heck, A., Introduction to Maple, 3rd edition (Springer-Verlag, New York, 2003).

[72] Homeier, H. H. H., "A Levin-type algorithm for accelerating the convergence of Fourier series," Numer. Algor. 3, 245 - 254 (1992).

[73] Homeier, H. H. H., "Some applications of nonlinear convergence accelerators," Int. J. Quantum Chem. 45, 545 - 562 (1993).

[74] Homeier, H. H. H., "Scalar Levin-type sequence transformations," J. Comput. Appl. Math. 122, 81 - 147 (2000). Reprinted in Brezinski, C. (Editor), Numerical Analysis 2000, Vol. 2: Interpolation and Extrapolation, 81 - 147 (Elsevier, Amsterdam, 2000).

[75] Homeier, H. H. H. and Weniger, E. J., "On remainder estimates for Levin-type sequence transformations," Comput. Phys. Commun. 92, 1 - 10 (1995).

[76] Ixaru, L. G., De Meyer, H., and Vanden Berghe, G., "Highly accurate eigenvalues for the distorted Coulomb potential," Phys. Rev. E 61, 3151 - 3159 (2000).

[77] Jentschura, U. D., Becher, J., Weniger, E. J., and Soff, G., "Resummation of QED perturbation series by sequence transformations and the prediction of perturbative coefficients," Phys. Rev. Lett. 85, 2446 - 2449 (2000).

[78] Jentschura, U. D., Gies, H., Valluri, S. R., Lamm, D., and Weniger, E., "QED effective action revisited," Can. J. Phys. 80, $267-284$ (2002).

[79] Jentschura, U. D., Mohr, P. J., and Soff, G., "Calculation of the electron self-energy for low nuclear charge," Phys. Rev. Lett. 82, 53 - 56 (1999).

[80] Jentschura, U. D., Mohr, P. J., and Soff, G., "Electron self-energy for the $K$ and $L$ shells at low nuclear charge," Phys. Rev. A 63, 042512-1 - 042512-19 (2001).

[81] Jentschura, U. D., Mohr, P. J., and Soff, G., "Calculation of QED effects in hydrogen," Hyperfine Interact. 132, $375-377$ (2001).

[82] Jentschura, U. D., Mohr, P. J., Soff, G., and Weniger, E. J., "Convergence acceleration via combined nonlinear-condensation transformations," Comput. Phys. Commun. 116, 28 - 54 (1999).

[83] Jentschura, U. D., Weniger, E. J., and Soff, G., "Asymptotic improvement of resummations and perturbative predictions in quantum field theory," J. Phys. G 26, 1545 - 1568 (2000).

[84] Jetzke, S. and Broad, J. T., "Evaluation of higher order matrix elements in atomic physics using convergence acceleration methods," Int. J. Mod. Phys. C 2, 377 - 382 (1991).

[85] Karliner, M., "Precise estimates of high orders in QCD," Acta Phys. Polon. B 29, $1505-1520$ (1998).

[86] King, F. W., "Convergence accelerator approach for the evaluation of some threeelectron integrals containing explicit $r_{i j}$ factors," Int. J. Quantum Chem. 72, 93 - 99 (1999).

[87] King, F. W., Smethells, G. J., Helleloid, G. T., and Pelzl, P. J., "Numerical evaluation of Hilbert transforms for oscillatory functions: A convergence accelerator approach," Comput. Phys. Commun. 145, 256 - 266 (2002). 
[88] Knopp, K., Theorie und Anwendung der unendlichen Reihen (Springer-Verlag, Berlin, 1964).

[89] Le Guillou, J. C. and Zinn-Justin, J. (Editors), Large-Order Behaviour of Perturbation Theory (North-Holland, Amsterdam, 1990).

[90] Levin, D., "Development of non-linear transformations for improving convergence of sequences," Int. J. Comput. Math. B 3, 371 - 388 (1973).

[91] Marchuk, G. I. and Shaidurov, V. V., Difference Methods and Their Extrapolations (Springer-Verlag, New York, 1983).

[92] Matos, A. C., "Linear difference operators and acceleration methods," IMA J. Numer. Anal. 20, 359 - 388 (2000).

[93] Neville, E. H., "Iterative interpolation," J. Indian Math. Soc. 20, 87 - 120 (1934).

[94] Nielsen, N., Die Gammafunktion (Chelsea, New York, 1965).

[95] Nörlund, N. E., Leçons sur les Séries d'Interpolation (Gautier-Villars, Paris, 1926).

[96] Nörlund, N. E., Vorlesungen über Differenzenrechnung (Chelsea, New York, 1954).

[97] Oleksy, C., "A convergence acceleration method for Fourier series," Comput. Phys. Commun. 96, $17-26$ (1996).

[98] Osada, N., "Extensions of Levin's transformation to vector sequences," Numer. Algor. 2, $121-132$ (1992).

[99] Padé, H., "Sur la représentation approachée d'une fonction par des fractions rationelles," Ann. Sci. Ec. Norm. Super. 9, 1 - 93 (1892).

[100] Pelzl, P. J. and King, F. W., "Convergence accelerator approach for the high-precision evaluation of three-electron correlated integrals," Phys. Rev. E 57, 7268 - 7273 (1998).

[101] Pelzl, P. J., Smethells, G. J., and King, F. W., "Improvements on the application of convergence accelerators for the evaluation of some three-electron atomic integrals," Phys. Rev. E 65, 036707-1-036707-11 (2002).

[102] Polly, R., Gruber, D., Windholz, L., Gleichmann, M. M., and Heß, B. A., "Relativistic all-electron ab initio calculations of CsHg potential energy curves including spin-orbit effects," J. Chem. Phys. 109, 9463 - 9472 (1998).

[103] Porras, I. and King, F. W., "Evaluation of some integrals for the atomic three-electron problem using convergence accelerators," Phys. Rev. A 49, 1637 - 1649 (1994).

[104] Richardson, L. F., "The deferred approach to the limit. I. Single lattice," Phil. Trans. Roy. Soc. London A 226, 299 - 349 (1927).

[105] Roy, D., Bhattacharya, R., and Bhowmick, S., "Rational approximants using the Levin $u$-transform: Application to the excluded volume-problem in polymers," Chem. Phys. Lett. 191, $609-613$ (1992).

[106] Roy, D., Bhattacharya, R., and Bhowmick, S., "Rational approximants generated by the $u$-transform," Comput. Phys. Commun. 78, 29 - 54 (1993).

[107] Roy, D., Bhattacharya, R., and Bhowmick, S., "Rational approximants using LevinWeniger transforms," Comput. Phys. Commun. 93, 159 - 178 (1996). 
[108] Roy, D., Bhattacharya, R., and Bhowmick, S., "Multipoint Levin-Weniger approximants and their application to the ground state energies of quantum anharmonic oscillators," Comput. Phys. Commun. 113, $131-144$ (1998).

[109] Samuel, M. A., Abraha, T., and Yu, J., "The strong coupling constant, $\alpha_{s}$, from $W$ + jet processes: An analysis using Padé approximants," Phys. Lett. B 394, 165 - 169 (1997).

[110] Samuel, M. A., Ellis, J., and Karliner, M., "Comparison of the Padé approximation method to perturbative QCD calculations," Phys. Rev. Lett. 74, 4380 - 4383 (1995).

[111] Samuel, M. A. and Li, G., "Estimating perturbative coefficients in quantum field theory and the ortho-positronium decay rate discrepancy," Phys. Lett. B 331, $114-$ 118 (1994).

[112] Samuel, M. A., Li, G., and Steinfelds, E., "Estimating perturbative coefficients in quantum field theory using Padé approximants," Phys. Rev. D 48, 869 - 872 (1993).

[113] Samuel, M. A., Li, G., and Steinfelds, E., "Estimating perturbative coefficients in quantum field theory using Padé approximants," Phys. Lett. B 323, 188 - 192 (1994).

[114] Samuel, M. A., Li, G., and Steinfelds, E., "Estimating perturbative coefficients in quantum field theory and statistical physics," Phys. Rev. E 51, 3911 - 3933 (1995).

[115] Samuel, M. A., Li, G., and Steinfelds, E., "Erratum: Estimating perturbative coefficients in quantum field theory and statistical physics," Phys. Rev. E 55, 2072 (1997).

[116] Sarkar, A., Haldar, S., Sen, D., and Roy, K. D., "Static local field factor for spin interacting system," Indian J. Phys. 75A, 29 - 32 (2001).

[117] Sarkar, A., Sen, D., Haldar, S., and Roy, D., "Static local field factor for dielectric screening function of electron gas at metallic and lower densities," Mod. Phys. Lett. B 12, $639-648$ (1998).

[118] Schnack, J., "Properties of the first excited state of nonbipartite Heisenberg spin rings," Phys. Rev. B 62, 14855 - 14859 (2000).

[119] Schneider, C., "Vereinfachte Rekursionen zur Richardson-Extrapolation in Spezialfällen," Numer. Math. 24, 177 - 184 (1975).

[120] Scott, T. C., Aubert-Frécon, M., and Andrae, D., "Asymptotics of quantum mechanical atom-ion systems," Appl. Anal. Engin. Commun. Comput. (AAECC) 13, 233 255 (2002).

[121] Shelef, R., New numerical quadrature formulas for Laplace transform inversion by Bromwich's integral, Master's thesis, Technion, Israel Institute of Technology, Haifa (1987). In Hebrew.

[122] Sidi, A., "A new method for deriving Padé approximants for some hypergeometric functions," J. Comput. Appl. Math. 7, 37 - 40 (1981).

[123] Sidi, A., "An algorithm for a special case of a generalization of the Richardson extrapolation process," Numer. Math. 38, 299 - 307 (1982).

[124] Sidi, A. and Levin, D., "Prediction properties of the $t$-transformation," SIAM J. Numer. Anal. 20, 589 - 598 (1983). 
[125] Simon, B., "Fifty years of eigenvalue perturbation theory," Bull. Amer. Math. Soc. 24, 303 - 319 (1991).

[126] Smith, D. A. and Ford, W. F., "Acceleration of linear and logarithmic convergence," SIAM J. Numer. Anal. 16, 223 - 240 (1979).

[127] Smith, D. A. and Ford, W. F., "Numerical comparisons of nonlinear convergence accelerators," Math. Comput. 38, $481-499$ (1982).

[128] Soff, G., Bednyakov, I., Beier, T., Erler, F., Goidenko, I. A., Jentschura, U. D., Labzowsky, L. N., Nefiodov, A. V., Plunien, G., Schützhold, R., and Zschocke, S., "Effects of QED and beyond from the atomic binding energy," Hyperfine Interact. 132, 75 - 103 (2001).

[129] Steele, T. G. and Elias, V., "Padé-improved extraction of $\alpha_{s}\left(M_{\tau}\right)$ from $R_{\tau}$," Mod. Phys. Lett. A 13, $3151-3159$ (1998).

[130] Steinborn, E. O. and Weniger, E. J., "Sequence transformations for the efficient evaluation of infinite series representations of some molecular integrals with exponentially decaying basis functions," J. Mol. Struct. (Theochem) 210, 71 - 78 (1990).

[131] Véniard, V. and Piraux, B., "Interaction of atomic hydrogen with an ultra-short laser pulse having a pedestal," Optics Commun. 84, 264 - 268 (1991).

[132] Weniger, E. J., "Nonlinear sequence transformations for the acceleration of convergence and the summation of divergent series," Comput. Phys. Rep. 10, 189 - 371 (1989).

[133] Weniger, E. J., "On the summation of some divergent hypergeometric series and related perturbation expansions," J. Comput. Appl. Math. 32, 291 - 300 (1990).

[134] Weniger, E. J., "Interpolation between sequence transformations," Numer. Algor. 3, $477-486$ (1992).

[135] Weniger, E. J., "On the efficiency of linear but nonregular sequence transformations," in Cuyt, A. (Editor), Nonlinear Numerical Methods and Rational Approximation II, 269 - 282 (Kluwer, Dordrecht, 1994).

[136] Weniger, E. J., Verallgemeinerte Summationsprozesse als numerische Hilfsmittel für quantenmechanische und quantenchemische Rechnungen, Habilitation thesis, Fachbereich Chemie und Pharmazie, Universität Regensburg (1994). Los Alamos Preprint math-ph/0306048 (http://arXiv.org).

[137] Weniger, E. J., "Nonlinear sequence transformations: A computational tool for quantum mechanical and quantum chemical calculations," Int. J. Quantum Chem. 57, 265 -280 (1996).

[138] Weniger, E. J., "Erratum: Nonlinear sequence transformations: A computational tool for quantum mechanical and quantum chemical calculations," Int. J. Quantum Chem. 58, 319 - 321 (1996).

[139] Weniger, E. J., "A convergent renormalized strong coupling perturbation expansion for the ground state energy of the quartic, sextic, and octic anharmonic oscillator," Ann. Phys. (NY) 246, 133 - 165 (1996).

[140] Weniger, E. J., "Computation of the Whittaker function of the second kind by summing its divergent asymptotic series with the help of nonlinear sequence transformations," Comput. Phys. 10, 496 - 503 (1996). 
[141] Weniger, E. J., "Construction of the strong coupling expansion for the ground state energy of the quartic, sextic and octic anharmonic oscillator via a renormalized strong coupling expansion," Phys. Rev. Lett. 77, 2859 - 2862 (1996).

[142] Weniger, E. J., "Performance of superconvergent perturbation theory," Phys. Rev. A 56, $5165-5168$ (1997).

[143] Weniger, E. J., "Prediction properties of Aitken's iterated $\Delta^{2}$ process, of Wynn's epsilon algorithm, and of Brezinski's iterated theta algorithm," J. Comput. Appl. Math. 329 - 356 (2000). Reprinted in Brezinski, C. (Editor), Numerical Analysis 2000, Vol. 2: Interpolation and Extrapolation, 329 - 356 (Elsevier, Amsterdam, 2000).

[144] Weniger, E. J., "Irregular input data in convergence acceleration and summation processes: General considerations and some special Gaussian hypergeometric series as model problems," Comput. Phys. Commun. 133, 202 - 228 (2001).

[145] Weniger, E. J., "A rational approximant for the digamma function," To appear in Numerical Algorithms (Proceedings of the International Conference on Numerical Algorithms, Marrakesh, Morocco, October 1 - 5, 2001) (2001). Los Alamos Preprint math.NA/0306305 (http://arXiv .org).

[146] Weniger, E. J., "Mathematical properties of a new Levin-type sequence transformation introduced by Čížek, Zamastil, and Skála. II. Convergence theory," (2003). In preparation (to be submitted to Journal of Mathematical Physics).

[147] Weniger, E. J. and Č́ížek, J., "Rational approximations for the modified Bessel function of the second kind," Comput. Phys. Commun. 59, 471 - 493 (1990).

[148] Weniger, E. J., Čížek, J., and Vinette, F., "Very accurate summation for the infinite coupling limit of the perturbation series expansions of anharmonic oscillators," Phys. Lett. A 156, 169 - 174 (1991).

[149] Weniger, E. J., Cížek, J., and Vinette, F., "The summation of the ordinary and renormalized perturbation series for the ground state energy of the quartic, sextic, and octic anharmonic oscillators using nonlinear sequence transformations," J. Math. Phys. 34, $571-609$ (1993).

[150] Weniger, E. J., Grotendorst, J., and Steinborn, E. O., "Some applications of nonlinear convergence accelerators," Int. J. Quantum Chem. Symp. 19, 181 - 191 (1986).

[151] Weniger, E. J. and Steinborn, E. O., "Nonlinear sequence transformations for the efficient evaluation of auxiliary functions for GTO molecular integrals," in Defranceschi, M. and Delhalle, J. (Editors), Numerical Determination of the Electronic Structure of Atoms, Diatomic and Polyatomic Molecules, 341 - 346 (Kluwer, Dordrecht, 1989).

[152] Wimp, J., Sequence Transformations and Their Applications (Academic Press, New York, 1981).

[153] Wynn, P., "On a device for computing the $e_{m}\left(S_{n}\right)$ transformation," Math. Tables Aids Comput. 10, 91 - 96 (1956). 